\title{
Prostatic urethral lift for the treatment of lower urinary tract symptoms in men with benign prostatic hyperplasia (Review)
}

Jung JH, Reddy B, McCutcheon KA, Borofsky M, Narayan V, Kim MH, Dahm P

Jung JH, Reddy B, McCutcheon KA, Borofsky M, Narayan V, Kim MH, Dahm P.

Prostatic urethral lift for the treatment of lower urinary tract symptoms in men with benign prostatic hyperplasia.

Cochrane Database of Systematic Reviews 2019, Issue 5. Art. No.: CD012832.

DOI: 10.1002/14651858.CD012832.pub2.

www.cochranelibrary.com

Prostatic urethral lift for the treatment of lower urinary tract symptoms in men with benign prostatic 
TABLE OF CONTENTS

ABSTRAC

PLAIN LANGUAGE SUMMARY

SUMMARY OF FINDINGS

BACKGROUND

OBJECTIVES

METHODS

RESULTS

Figure 1.

Figure 2.

Figure 3.

DISCUSSION

AUTHORS' CONCLUSIONS

ACKNOWLEDGEMENTS

REFERENCES

CHARACTERISTICS OF STUDIES

DATA AND ANALYSES

Analysis 1.1. Comparison 1 Prostatic urethral lift (PUL) versus sham (short term), Outcome 1 Urological symptom scores. ......

Analysis 1.2. Comparison 1 Prostatic urethral lift (PUL) versus sham (short term), Outcome 2 Quality of life.

Analysis 1.3. Comparison 1 Prostatic urethral lift (PUL) versus sham (short term), Outcome 3 Major adverse events. ...............

Analysis 1.4. Comparison 1 Prostatic urethral lift (PUL) versus sham (short term), Outcome 4 Ejaculatory function. .................

Analysis 1.5. Comparison 1 Prostatic urethral lift (PUL) versus sham (short term), Outcome 5 Erectile function. ......................

Analysis 1.6. Comparison 1 Prostatic urethral lift (PUL) versus sham (short term), Outcome 6 Minor adverse events. ...............

Analysis 1.7. Comparison 1 Prostatic urethral lift (PUL) versus sham (short term), Outcome 7 Acute urinary retention. ............

Analysis 1.8. Comparison 1 Prostatic urethral lift (PUL) versus sham (short term), Outcome 8 Hospital stay (days).

Analysis 2.1. Comparison 2 Prostatic urethral lift (PUL) versus transurethral resection of prostate (TURP) (short term), Outcome 1 Urological symptom scores.

Analysis 2.2. Comparison 2 Prostatic urethral lift (PUL) versus transurethral resection of prostate (TURP) (short term), Outcome 2 Quality of life.

Analysis 2.3. Comparison 2 Prostatic urethral lift (PUL) versus transurethral resection of prostate (TURP) (short term), Outcome 3 Major adverse events.

Analysis 2.4. Comparison 2 Prostatic urethral lift (PUL) versus transurethral resection of prostate (TURP) (short term), Outcome 4 Retreatment.

Analysis 2.5. Comparison 2 Prostatic urethral lift (PUL) versus transurethral resection of prostate (TURP) (short term), Outcome 5 Erectile function.

Analysis 2.6. Comparison 2 Prostatic urethral lift (PUL) versus transurethral resection of prostate (TURP) (short term), Outcome 6 Ejaculatory function.

Analysis 2.7. Comparison 2 Prostatic urethral lift (PUL) versus transurethral resection of prostate (TURP) (short term), Outcome 7 Minor adverse events.

Analysis 2.8. Comparison 2 Prostatic urethral lift (PUL) versus transurethral resection of prostate (TURP) (short term), Outcome 8 Acute urinary retention.

Analysis 2.9. Comparison 2 Prostatic urethral lift (PUL) versus transurethral resection of prostate (TURP) (short term), Outcome 9 Indwelling urinary catheter.

Analysis 2.10. Comparison 2 Prostatic urethral lift (PUL) versus transurethral resection of prostate (TURP) (short term), Outcome 10 Hospital stay (days).

Analysis 3.1. Comparison 3 Prostatic urethral lift (PUL) versus transurethral resection of prostate (TURP) (long term), Outcome 1 Urological symptom scores.

Analysis 3.2. Comparison 3 Prostatic urethral lift (PUL) versus transurethral resection of prostate (TURP) (long term), Outcome 2 Quality of life.

Analysis 3.3. Comparison 3 Prostatic urethral lift (PUL) versus transurethral resection of prostate (TURP) (long term), Outcome 3 Retreatment.

Analysis 3.4. Comparison 3 Prostatic urethral lift (PUL) versus transurethral resection of prostate (TURP) (long term), Outcome 4 Erectile function. 
Analysis 3.5. Comparison 3 Prostatic urethral lift (PUL) versus transurethral resection of prostate (TURP) (long term), Outcome 5 Ejaculatory function.

Analysis 3.6. Comparison 3 Prostatic urethral lift (PUL) versus transurethral resection of prostate (TURP) (long term), Outcome

6 Minor adverse events.

ADDITIONAL TABLES

APPENDICES 
[Intervention Review]

\section{Prostatic urethral lift for the treatment of lower urinary tract symptoms in men with benign prostatic hyperplasia}

Jae Hung Jung1,2, Balaji Reddy³, Karen Ann McCutcheon4, Michael Borofsky5, Vikram Narayan5, Myung Ha Kim6, Philipp Dahm5,7

1Department of Urology, Yonsei University Wonju College of Medicine, Wonju, Korea, South. 2Institute of Evidence Based Medicine, Yonsei University Wonju College of Medicine, Wonju, Korea, South. ${ }^{3}$ Department of Urology, Massachusetts General Hospital, Boston, Massachusetts, USA. ${ }^{4}$ School of Nursing and Midwifery, Queen's University Belfast, Belfast, UK. ${ }^{5}$ Department of Urology, University of Minnesota, Minneapolis, Minnesota, USA. ${ }^{6}$ Yonsei Wonju Medical Library, Yonsei University Wonju College of Medicine, Wonju, Korea, South. ${ }^{7}$ Urology Section, Minneapolis VA Health Care System, Minneapolis, Minnesota, USA

Contact: Jae Hung Jung, Department of Urology, Yonsei University Wonju College of Medicine, 20 Ilsan-ro, Wonju, Gangwon, 26426, Korea, South.jungx563@gmail.com,geneuro95@yonsei.ac.kr.

Editorial group: Cochrane Urology Group.

Publication status and date: New, published in Issue 5, 2019.

Citation: Jung JH, Reddy B, McCutcheon KA, Borofsky M, Narayan V, Kim MH, Dahm P. Prostatic urethral lift for the treatment of lower urinary tract symptoms in men with benign prostatic hyperplasia. Cochrane Database of Systematic Reviews 2019, Issue 5. Art. No.: CD012832. DOI: 10.1002/14651858.CD012832.pub2.

Copyright @ 2019 The Cochrane Collaboration. Published by John Wiley \& Sons, Ltd.

\section{A B S T R A C T}

\section{Background}

A variety of minimally invasive surgical approaches are available as an alternative to transurethral resection of prostate (TURP) for the management of lower urinary tract symptoms (LUTS) in men with benign prostatic hyperplasia (BPH). A recent addition to these is prostatic urethral lift (PUL).

\section{Objectives}

To assess the effects of PUL for the treatment of LUTS in men with BPH.

\section{Search methods}

We performed a comprehensive search of multiple databases (the Cochrane Library, MEDLINE, Embase, LILACS, Scopus, Web of Science, and Google Scholar), trials registries, other sources of grey literature, and conference proceedings with no restrictions on the language of publication or publication status up until 31 January 2019.

\section{Selection criteria}

We included parallel group randomized controlled trials (RCTs). While we planned to include non-RCTs if RCTs had provided low-certainty evidence for a given outcome and comparison, we could not find any non-RCTs.

\section{Data collection and analysis}

Two review authors independently screened the literature, extracted data, and assessed risk of bias. We performed statistical analyses using a random-effects model and interpreted them according to the Cochrane Handbook for Systematic Reviews of Interventions. We planned subgroup analyses by age, prostate volume, and severity of baseline symptoms. We used the GRADE approach to rate the certainty of the evidence.

\section{Main results}

We included two RCTs with 297 participants comparing PUL to sham surgery or TURP. The mean age was 65.6 years and mean International Prostate Symptom Score was 22.7. Mean prostate volume was $42.2 \mathrm{~mL}$. We considered review outcomes measured up to and including 12

Prostatic urethral lift for the treatment of lower urinary tract symptoms in men with benign prostatic hyperplasia (Review) 
months after randomization as short-term and later than 12 months as long-term. For patient-reported outcomes, lower scores indicate more urological symptom improvement and higher quality of life. In contrast, higher scores refers to better erectile and ejaculatory function.

PUL versus sham: based on one study of 206 randomized participants with short follow-up (up to three months), PUL may lead to a clinically important improvement in urological symptom scores (mean difference (MD) -5.20, 95\% confidence interval (Cl) - 7.44 to -2.96; low-certainty evidence) and likely improves quality of life (MD $-1.20,95 \% \mathrm{Cl}-1.67$ to -0.73 ; moderate-certainty evidence). We are uncertain whether PUL increases major adverse events (very low-certainty evidence). There were no retreatments reported in either study group by three months. PUL likely results in little to no difference in erectile function (MD $-1.40,95 \% \mathrm{Cl}-3.24$ to 0.44 ; moderate-certainty evidence) and ejaculatory function (MD $0.50,95 \% \mathrm{Cl}-0.38$ to 1.38 ; moderate-certainty evidence).

PUL versus TURP: based on one study of 91 randomized participants with a short follow-up (up to 12 months), PUL may result in a substantially lesser improvement in urological symptom scores than TURP (MD 4.50, 95\% CI 1.10 to 7.90; low-certainty evidence). PUL may result in a slightly reduced or similar quality of life ( $\mathrm{MD} 0.30,95 \% \mathrm{Cl}-0.49$ to 1.09 ; low-certainty evidence). We are very uncertain whether PUL may cause fewer major adverse events but increased retreatments (both very low-certainty evidence). PUL probably results in little to no difference in erectile function (MD $0.80,95 \% \mathrm{Cl}-1.50$ to 3.10 ; moderate-certainty evidence), but probably results in substantially better ejaculatory function (MD 5.00, 95\% $\mathrm{Cl} 3.08$ to 6.92; moderate-certainty evidence).

With regards to longer term follow-up (up to 24 months) based on one study of 91 randomized participants, PUL may result in a substantially lesser improvement in urological symptom score (MD 6.10,95\% Cl 2.16 to 10.04 ; low-certainty evidence) and result in little worse to no difference in quality of life (MD $0.80,95 \% \mathrm{Cl} 0.00$ to 1.60 ; low-certainty evidence). The study did not report on major adverse events. We are very uncertain whether PUL increases retreatment (very low-certainty evidence). PUL likely results in little to no difference in erectile function (MD 1.60, 95\% Cl-0.80 to 4.00; moderate-certainty evidence), but may result in substantially better ejaculatory function (MD 4.30, $95 \% \mathrm{Cl} 2.17$ to 6.43 ; low-certainty evidence).

We were unable to perform any of the predefined secondary analyses for either comparison.

We found no evidence for other comparisons such as PUL versus laser ablation or enucleation.

\section{Authors' conclusions}

PUL appears less effective than TURP in improving urological symptoms both short-term and long term, while quality of life outcomes may be similar. The effect on erectile function appears similar but ejaculatory function may be better. We are uncertain about major adverse events short-term and found no long-term information. We are very uncertain about retreatment rates both short-term and long-term. We were unable to assess the effects of PUL in subgroups based on age, prostate size, or symptom severity and also could not assess how PUL compared to other surgical management approaches. Given the large numbers of alternative treatment modalities to treat men with LUTS secondary to $\mathrm{BPH}$, this represents important information that should be shared with men considering surgical treatment.

\section{PLAIN LANGUAGE SUMMARY}

\section{Prostatic urethral lift for the treatment of lower urinary tract symptoms in men with benign prostatic hyperplasia}

\section{Review question}

Does prostatic urethral lift (PUL) improve bothersome urinary symptoms without unwanted side effects in men with an enlarged prostate?

\section{Background}

Prostate enlargement is common in men as they get older and may cause difficulty with urination. PUL is a new procedure to relieve urinary symptoms such as having to urinate often, having to strain or not being able to empty the bladder completely. Unwanted side effects of treatment may be problems with erections, ejaculation or needing to be treated again. PUL works by placing little hooks that compress the tissue of the prostate to open up the urinary stream without the need to cut or remove any tissue. We did this review to compare PUL to other surgical treatments in men with an enlarged prostate and bothersome urinary symptoms.

\section{Study characteristics}

We included two randomized controlled studies (clinical trials where people are randomly put into one of two or more treatment groups) with 297 men comparing PUL to sham surgery (participants are made to believe they received treatment, while in reality they did not) or transurethral resection of prostate (TURP: removing the excess prostate growth using a camera and an electrically activated resecting loop inserted via the penis). The average age of the participants was 65.6 years.

\section{Key results}


Compared to sham surgery up to three months, PUL may improve urinary symptoms and likely improves quality of life without additional unwanted side effects after surgery. In the short term, there were no additional surgeries because PUL did not work. PUL likely does not make erections or ejaculation worse.

Compared to TURP up to 24 months, PUL may be less effective in relieving urinary symptoms, but result in similar quality of life. PUL may preserve ejaculation, but may have less unwanted effects on erections than TURP. However, we are either very uncertain or have no evidence about serious unwanted side effects or the need for additional treatment after surgery.

Findings of this review are up-to-date until 31 January, 2019.

\section{Certainty of the evidence}

The certainty of evidence for most outcomes was low. This means that the true effect may be substantially different from what this review shows. 


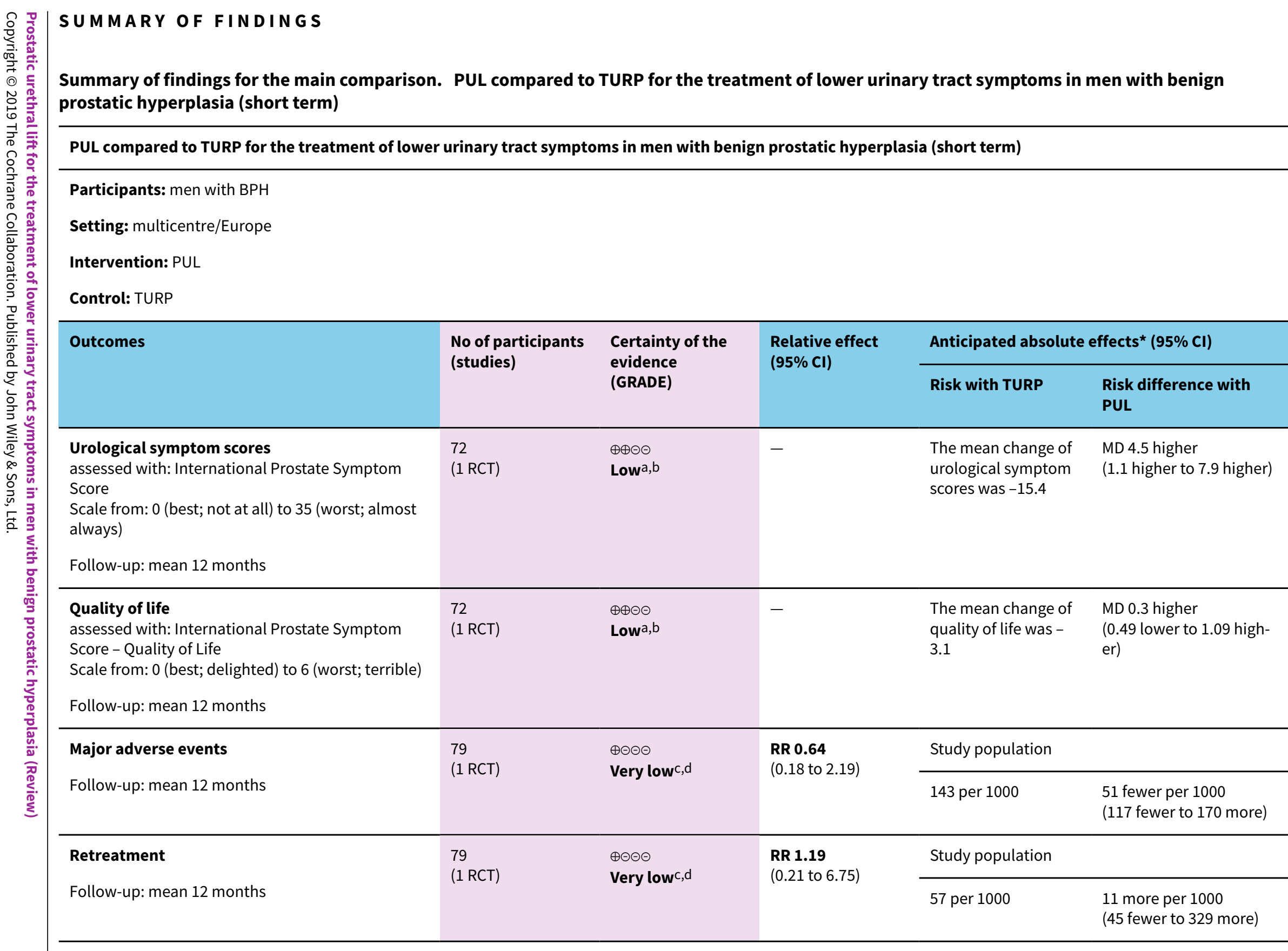




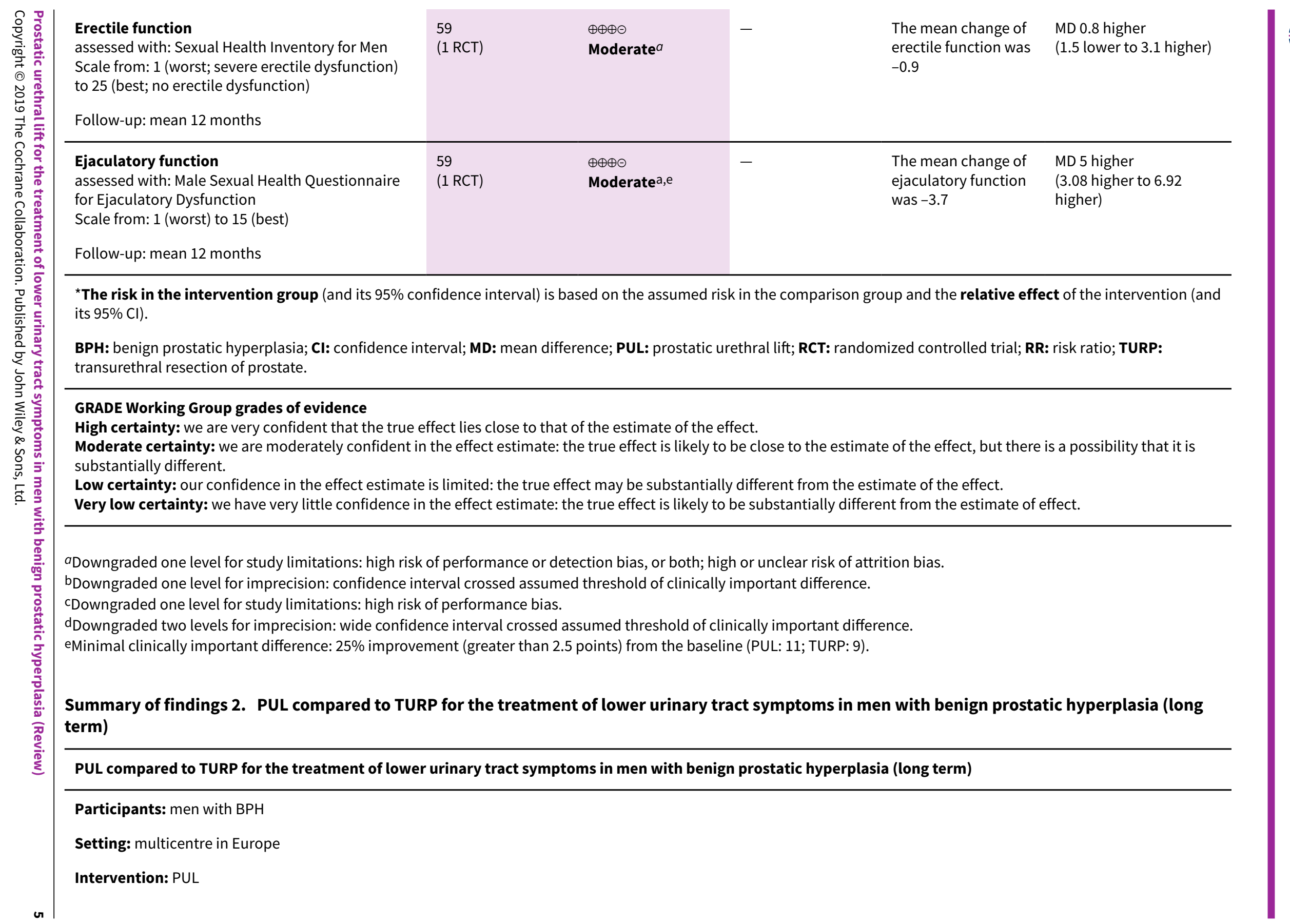




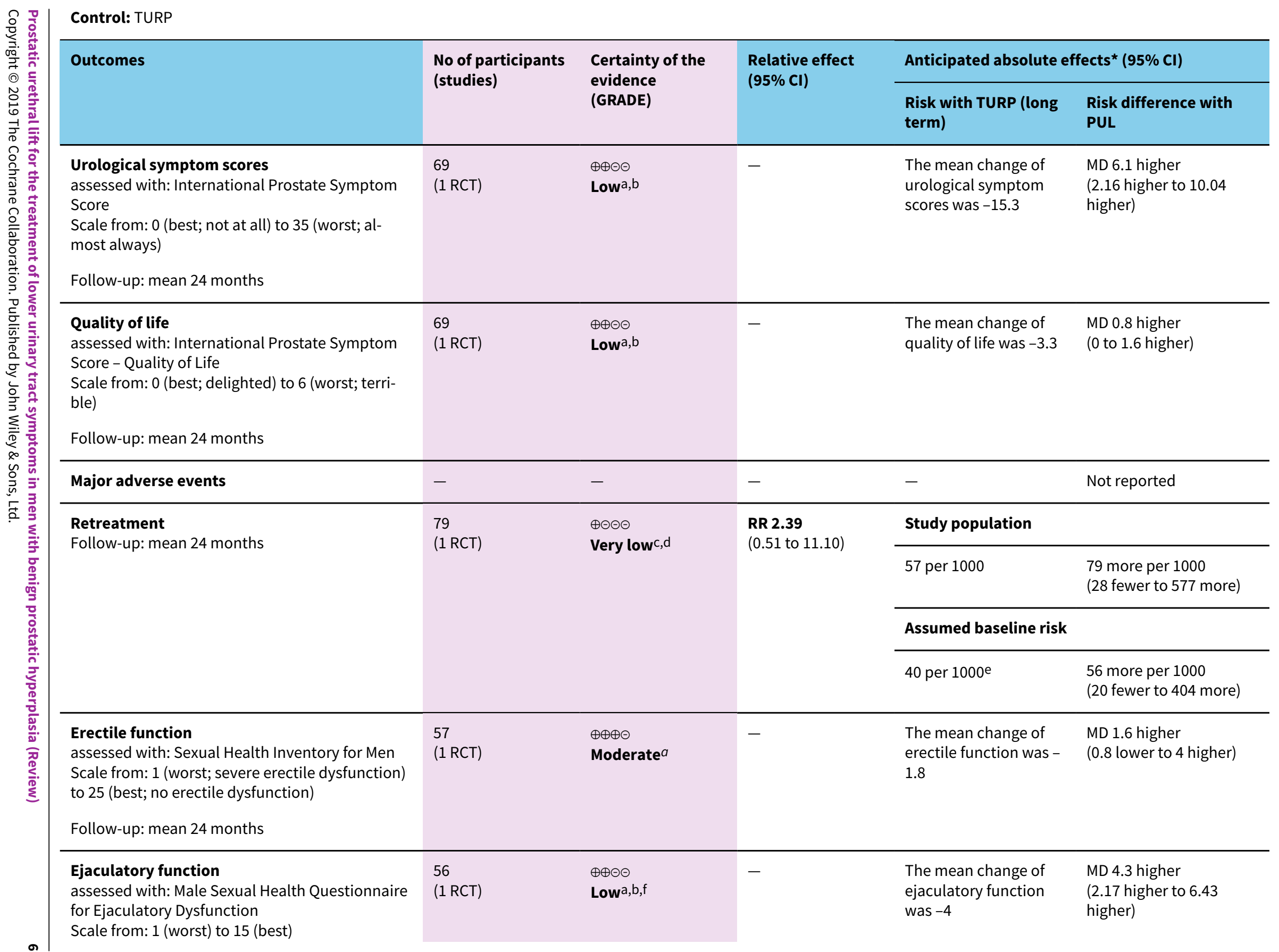


*The risk in the intervention group (and its $95 \%$ confidence interval) is based on the assumed risk in the comparison group and the relative effect of the intervention (and its $95 \% \mathrm{Cl})$.

BPH: benign prostatic hyperplasia; CI: confidence interval; MD: mean difference; PUL: prostatic urethral lift; RCT: randomized controlled trial; RR: risk ratio; TURP: transurethral resection of prostate.

\section{GRADE Working Group grades of evidence}

High certainty: we are very confident that the true effect lies close to that of the estimate of the effect

Moderate certainty: we are moderately confident in the effect estimate: the true effect is likely to be close to the estimate of the effect, but there is a possibility that it is substantially different

Low certainty: our confidence in the effect estimate is limited: the true effect may be substantially different from the estimate of the effect

Very low certainty: we have very little confidence in the effect estimate: the true effect is likely to be substantially different from the estimate of effect

aDowngraded one level for study limitations: high risk of performance or detection bias, or both; high or unclear risk of attrition bias.

bDowngraded one level for imprecision: confidence interval crossed assumed threshold of clinically important difference.

cDowngraded one level for study limitations: high risk of performance bias.

dDowngraded two level for imprecision: wide confidence interval crossed assumed threshold of clinically important difference.

eEstimates for control event rates for retreatment come from Strope 2015.

fMinimal clinically important difference: $25 \%$ improvement (greater than 2.5 points) from the baseline (PUL: 11; TURP: 9).

\section{Summary of findings 3. PUL compared to sham for the treatment of lower urinary tract symptoms in men with benign prostatic hyperplasia (short} term)

\section{PUL compared to sham for the treatment of lower urinary tract symptoms in men with benign prostatic hyperplasia (short term)}

\section{Participants: men with $\mathrm{BPH}$}

Setting: multicentre in US, Canada, and Australia

Intervention: PUL

Control: sham

\begin{tabular}{|c|c|c|c|c|c|}
\hline \multirow[t]{2}{*}{ Outcomes } & \multirow{2}{*}{$\begin{array}{l}\text { No of participants } \\
\text { (studies) }\end{array}$} & \multirow{2}{*}{$\begin{array}{l}\text { Certainty of the } \\
\text { evidence } \\
\text { (GRADE) }\end{array}$} & \multirow{2}{*}{$\begin{array}{l}\text { Relative effect } \\
(95 \% \mathrm{CI})\end{array}$} & \multicolumn{2}{|c|}{ Anticipated absolute effects ${ }^{\star}(95 \% \mathrm{Cl})$} \\
\hline & & & & $\begin{array}{l}\text { Risk with sham } \\
\text { (short term) }\end{array}$ & $\begin{array}{l}\text { Risk difference with } \\
\text { PUL }\end{array}$ \\
\hline $\begin{array}{l}\text { Urological symptom scores } \\
\text { assessed with: International Prostate Symptom Score: } \\
0 \text { (best; not at all) to } 35 \text { (worst; almost always) }\end{array}$ & $\begin{array}{l}206 \\
(1 \mathrm{RCT})\end{array}$ & $\begin{array}{l}\oplus \oplus \ominus \ominus \\
\text { Low }{ }^{a, b}\end{array}$ & - & $\begin{array}{l}\text { The mean change of } \\
\text { urological symptom } \\
\text { scores was }-5.9\end{array}$ & $\begin{array}{l}\text { MD } 5.2 \text { lower } \\
\text { ( } 7.44 \text { lower to } 2.96 \\
\text { lower) }\end{array}$ \\
\hline
\end{tabular}




\begin{tabular}{|c|c|c|c|c|c|c|}
\hline \multirow{2}{*}{\multicolumn{2}{|c|}{ 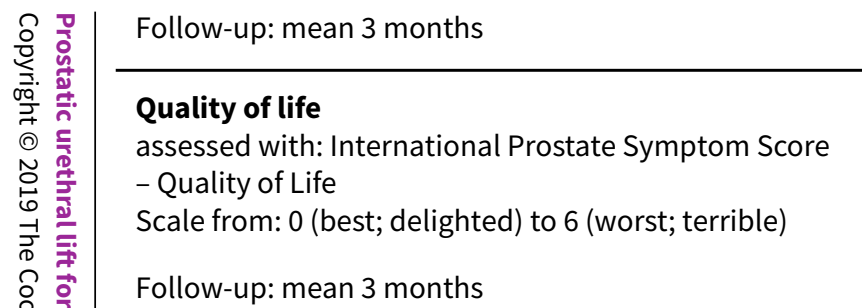 }} & & & \multirow[b]{2}{*}{$\begin{array}{l}\text { MD } 1.2 \text { lower } \\
\text { (1.67 lower to } 0.73 \\
\text { lower) }\end{array}$} \\
\hline & & $\begin{array}{l}206 \\
(1 \mathrm{RCT})\end{array}$ & $\begin{array}{l}\oplus \oplus \oplus \ominus \\
\text { Moderate } a\end{array}$ & - & $\begin{array}{l}\text { The mean change of } \\
\text { quality of life was }-1\end{array}$ & \\
\hline 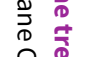 & Major adverse events & \multirow{2}{*}{$\begin{array}{l}206 \\
(1 \mathrm{RCT})\end{array}$} & \multirow{2}{*}{$\begin{array}{l}\oplus \ominus \ominus \ominus \\
\text { Very lowa,c }\end{array}$} & \multirow{2}{*}{$\begin{array}{l}\text { RR } 3.30 \\
\text { (0.41 to } 26.28)\end{array}$} & \multicolumn{2}{|l|}{ Study population } \\
\hline 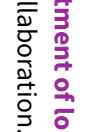 & & & & & 15 per 1000 & $\begin{array}{l}35 \text { more per } 1000 \\
\text { ( } 9 \text { fewer to } 383 \\
\text { more) }\end{array}$ \\
\hline 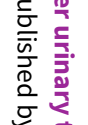 & $\begin{array}{l}\text { Retreatment } \\
\text { Follow-up: mean } 3 \text { months }\end{array}$ & $\begin{array}{l}206 \\
(1 \mathrm{RCT})\end{array}$ & $\begin{array}{l}\oplus \oplus \ominus \ominus \\
\text { Low }\end{array}$ & Not estimable & - & - \\
\hline 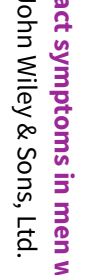 & $\begin{array}{l}\text { Erectile function } \\
\text { assessed with: International Index of Erectile Func- } \\
\text { tion-5 } \\
\text { Scale from: } 1 \text { (worst; severe erectile dysfunction) to } 25 \\
\text { (best; no erectile dysfunction) } \\
\text { Follow-up: mean } 3 \text { months }\end{array}$ & $\begin{array}{l}197 \\
(1 \mathrm{RCT})\end{array}$ & $\begin{array}{l}\oplus \oplus \oplus \ominus \\
\text { Moderate }^{a}\end{array}$ & - & $\begin{array}{l}\text { The mean change of } \\
\text { erectile function was } \\
1.5\end{array}$ & $\begin{array}{l}\text { MD } 1.4 \text { lower } \\
\text { (3.24 lower to } 0.44 \\
\text { higher) }\end{array}$ \\
\hline 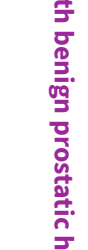 & $\begin{array}{l}\text { Ejaculatory function } \\
\text { assessed with: Male Sexual Health Questionnaire for } \\
\text { Ejaculatory Dysfunction } \\
\text { Scale from: } 1 \text { (worst) to } 15 \text { (best) } \\
\text { Follow-up: mean } 3 \text { months }\end{array}$ & $\begin{array}{l}144 \\
(1 \mathrm{RCT})\end{array}$ & $\begin{array}{l}\oplus \oplus \oplus \ominus \\
\text { Moderate } e, f\end{array}$ & - & $\begin{array}{l}\text { The mean change of } \\
\text { ejaculatory function } \\
\text { was } 1.7\end{array}$ & $\begin{array}{l}\text { MD } 0.5 \text { higher } \\
\text { (0.38 lower to } 1.38 \\
\text { higher) }\end{array}$ \\
\hline 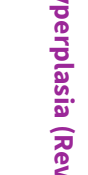 & \multicolumn{6}{|c|}{$\begin{array}{l}{ }^{\star} \text { The risk in the intervention group (and its } 95 \% \text { confidence interval) is based on the assumed risk in the comparison group and the relative effect of the intervention (and } \\
\text { its } 95 \% \mathrm{Cl} \text { ). }\end{array}$} \\
\hline & \multicolumn{6}{|c|}{$\begin{array}{l}\text { GRADE Working Group grades of evidence } \\
\text { High certainty: we are very confident that the true effect lies close to that of the estimate of the effect. } \\
\text { Moderate certainty: we are moderately confident in the effect estimate: the true effect is likely to be close to the estimate of the effect, but there is a possibility that it is } \\
\text { substantially different. } \\
\text { Low certainty: our confidence in the effect estimate is limited: the true effect may be substantially different from the estimate of the effect. } \\
\text { Very low certainty: we have very little confidence in the effect estimate: the true effect is likely to be substantially different from the estimate of effect. }\end{array}$} \\
\hline
\end{tabular}


Downgraded one level for study limitations: high risk of performance.

bDowngraded one level for imprecision: confidence interval crossed assumed threshold of clinically important difference.

cDowngraded two levels for imprecision: wide confidence interval crossed assumed threshold of clinically important difference.

dDowngraded one level for imprecision: very rare event.

eDowngraded one level for study limitations: high risk of performance or attrition bias, or both.

fMinimal clinically important difference: $25 \%$ improvement (greater than 2 points) from the baseline (PUL: 8.7; sham: 8.8). 


\section{B A C K G R O U N D}

\section{Description of the condition}

The prostate gland is an organ approximately the size of a walnut that is located below the urinary bladder encircling the urethra (Leissner 1979). Benign prostatic hyperplasia (BPH) is a histological diagnosis defined as an increased number of epithelial and stromal cells in the prostate; this may cause prostatic enlargement and subsequently compression of the urethra and obstruction (Roehrborn 2008a). Therefore, BPH may develop with or without lower urinary tract symptoms (LUTS) in men over the age of 40 years (Dunphy 2015). BPH receives clinical significance when associated with bothersome LUTS (Roehrborn 2008a). Symptom bother typically correlates with the increased number and severity of symptoms, which relates to both quality of life impairment and treatment seeking (Agarwal 2014). Self-administered questionnaires, namely International Prostate Symptom Score (IPSS), include the quality of life domain to evaluate the relative degree of bother across all LUTS (Barry 1995). Chapple 2017 reported that increasing LUTS severity was associated with worsening men's overall distress using patient perception of bladder condition which is a single-item global question (ranging from 1 (causes no problems at all) to 6 (causes severe problems)). In this Cochrane Review, we considered the term $\mathrm{BPH}$ as prostatic enlargement with LUTS through which to define the disease condition and potential need for intervention.

BPH can progress and cause serious consequences such as acute urinary retention, urinary tract infection, and upper urinary tract deterioration. $\mathrm{BPH}$ also results in a negative impact on public health and a reduction in a person's quality of life (Kozminski 2015; Martin 2014). In Europe, 30\% of men over 50 years of age, equivalent to 26 million men, are affected by bothersome LUTS, including storage symptoms (such as urinary frequency, urgency, and nocturia) or voiding symptoms (such as urinary hesitancy, weak urinary stream, straining to void, and prolonged voiding), or both. The yearly reported associated number of medical prescriptions is estimated to be around 11.6 million for 74 million people at risk from 2004 to 2008 (Cornu 2010). The prevalence of LUTS, according to an international study involving 7588 men was $18 \%$ in the ages of 40 s, $29 \%$ in the 50 s, $40 \%$ in the 60 s, and $56 \%$ in the 70 s (Homma 1997). In the USA, an estimated eight million men older than 50 years of age have BPH (Roehrborn 2008b). More recent data showed that the lifetime prevalence of BPH was $26.2 \%$ (95\% Cl: 22.8-29.6\%) (Lee 2017).

\section{Diagnosis}

Initial evaluation of LUTS suggestive of BPH includes patient history, physical examination including a digital rectal examination, urinalysis, prostate-specific antigen (PSA) blood test, voiding diary, and IPSS (EAU 2017; McVary 2011). A digital rectal examination is performed to assess the prostate for size, and for any lesions suspicious for cancer. The PSA is secreted by the prostate gland and is found to be abnormally elevated in conditions such as prostate cancer, BPH, infection, or inflammation of the prostate (EAU 2017; McVary 2011). The IPSS is used to assess urinary symptom severity and quality of life. It is also used to document subjective responses to treatment (Barry 1992; EAU 2017; McVary 2011). Measurement of maximum flow rate $\left(Q_{\max }\right)$ and postvoid residual (PVR) are also often used in diagnosis and treatment decisions (EAU 2017; McVary 2011). A low $\mathrm{Q}_{\max }$ and a large PVR predict an increased risk of symptom progression (Crawford 2006). Other tests include radiological imaging, urodynamic evaluation, and cystoscopy to further determine appropriate treatment and predict treatment response (Egan 2016; McVary 2011).

\section{Treatment}

Treatment decisions are based on symptoms and the degree of bother noted by the patient. Initial treatment options for $\mathrm{BPH}$ include conservative management (watchful waiting and lifestyle modification) and medication (alpha blockers and 5alpha reductase inhibitors) (EAU 2017; McVary 2011). If patients have been refractory to conservative and medical treatment, or $\mathrm{BPH}$ causes subsequent complications, such as acute urinary retention, recurrent urinary tract infection, bladder stones or diverticula, haematuria, or renal insufficiency, surgical options are considered (EAU 2017; McVary 2011). Until the 1970s, the only option available to treat this condition and relieve LUTS was an open or endoscopic surgery with the aim of removing or resecting prostatic tissue to open up the blocked urethra (Pariser 2015). Clinical guidelines recommend monopolar or bipolar transurethral resection of the prostate (TURP) as a standard treatment modality in regards to subjective symptom relief and objective improvements in urinary flow (EAU 2017; McVary 2011), but this procedure is also associated with significant morbidity and long-term complications, including haematuria requiring blood transfusion, urethral stricture, recurrent urinary tract infection, and urinary incontinence. Moreover, men may experience ejaculatory (65\%) and erectile dysfunction (10\%) related to TURP (Roehrborn 2003). Furthermore, BPH is a disease common in elderly men who have an increased risk of complications for general anaesthesia and the surgery itself (Dunphy 2015; Yoo 2012). Nowadays, other minimally invasive surgeries using electrode, laser, transurethral thermal ablation of the prostate (needle ablation, microwave therapy, and radiofrequency ablative techniques), and mechanical stents have been developed as alternatives to TURP (EAU 2017; McVary 2011). While new laser-based procedures have demonstrated a decrease in short-term complications, such as bleeding, they also have similar adverse effects on sexual function when compared with TURP (NICE 2015). Thermal ablation therapies may have fewer serious adverse events than TURP, but can be associated with long periods of irritative symptoms and the need of urinary catheterization (EAU 2017; McVary 2011). In addition, most men considering surgical intervention do so in the expectation of this providing a more definitive therapy for their LUTS thereby precluding the need for additional medical or surgical therapy. Given relatively high rates of reoperation or continued use of medical therapy after surgical treatment (or both), there are concerns about the durability of newly launched minimal invasive surgeries (NICE 2015; Strope 2015).

\section{Description of the intervention}

A less invasive surgical intervention known as the prostatic urethral lift (PUL) has recently become available. The US Food and Drug Administration approved PUL in September 2013 and the National Institute for Health and Care Excellence in the UK in September 2015 (McNicholas 2016). As the PUL procedure can be performed under local anaesthesia with oral or intravenous sedation, and also be performed in men with blood clotting disorders or men receiving anticoagulant therapy, it is more suitable for men at high risk of general anaesthesia (Chin 2012; Woo 2012). Typical inclusion criteria of PUL are a prostate volume between $20 \mathrm{~mL}$ and $70 \mathrm{~mL}$, 
IPSS of 12 or greater, a measured $\mathrm{Q}_{\max }$ of $15 \mathrm{~mL} /$ second or less, and PVR of less than $350 \mathrm{~mL}$ (McNicholas 2016). The PUL system consists of two single-use components (delivery device and an implant). The delivery device consists of a hand-held pistol grip to which a needle-shaped probe is attached. Each PUL implant consists of a super-elastic nitinol capsular tab, a polyethylene terephthalate monofilament, and a stainless-steel urethral end piece. The surgeon inserts the probe into the urethra until it reaches the prostatic urethra (the widest part of the prostatic urethra); a fine needle at the end of the probe deploys and secures an implant in a lobe of the prostate (McNicholas 2016). One end of the implant is anchored in the urethra and the other is attached to the firm outer surface of the prostatic capsule, so pulling the prostatic lobe away from the urethra. This is repeated on the other lobe of the prostate. Systematically, four implants for PUL are delivered, two each the right and left lateral lobes of the prostate (at the 2 and 10 o'clock position, distally from approximately $1.5 \mathrm{~cm}$ distal to the bladder neck). PUL is unable to treat a median lobe of the prostate which causes obstructive intravesical protrusion of prostate (McNicholas 2016).

\section{Adverse events of the intervention}

Mild adverse events, such as transient dysuria and haematuria are commonly reported with PUL (Chin 2012; Woo 2012). Incontinence was less prevalent with PUL (5\%) compared with TURP (11\%) (NICE 2015). However, reoperation rates were higher with the PUL (8\%) than with TURP (6\%) (NICE 2015). In one feasibility study, implant encrustation occurred when PUL implants are placed too close to the bladder and exposed to static urine (Chin 2012; Woo 2012).

\section{How the intervention might work}

The fundamental idea of PUL is the separation and distraction of the enlarged prostatic tissue by a series of implants. The PUL system uses adjustable, permanent implants to hold excess prostatic tissue out of the way and thereby open the narrowed urethra without cutting or removing enlarged prostatic tissue (McNicholas 2016). These implants are shaped as a double-ended hook, and aim to increase the opening of the urethra (McNicholas 2016).

\section{Why it is important to do this review}

Until now, it is unclear whether PUL actually translates into more clinical benefits and fewer adverse events in clinical practice. While there are existing systematic reviews that compare PUL to other therapies used to treat BPH (Jones 2016; Perera 2015; Sanchez-Gomez 2015), these reviews merely pooled efficacy measurements, such as IPSS, $\mathrm{Q}_{\max }$, and PVR from randomized controlled trials (RCTs) or non-RCTs, without discussing the safety of the intervention or assessing the certainty of evidence. No systematic review to date has used the same rigorous methodology as a Cochrane Review, which includes the application of the GRADE approach and its focus on patient-important outcomes (Guyatt 2008). In contemporary practice, with the availability of numerous minimally invasive procedures to treat $\mathrm{BPH}$, the findings of this Cochrane Review are expected to be relevant to policymakers, healthcare providers, and patients.

\section{OB JECTIVES}

To assess the effects of PUL for the treatment of LUTS in men with $\mathrm{BPH}$.

\section{METHODS}

\section{Criteria for considering studies for this review}

\section{Types of studies}

We included parallel-group RCTs and cluster-RCTs. We excluded cross-over trials, as these study designs are not relevant in this setting. If we had only found RCTs that provided low-certainty evidence for a given outcome and comparison, we planned to include non-RCTs, such as cohort and cross-sectional studies with concurrent comparison groups, as a source of complementary, sequential, or replacement evidence for RCTs (Schünemann 2013). We planned to exclude single-armed studies. We included studies regardless of their publication status or language of publication.

\section{Types of participants}

We defined the eligible patient population as men over the age of 40 years with a prostate volume of $20 \mathrm{~mL}$ or greater (as assessed by digital rectal examination, ultrasound or cross-sectional imaging, or both), with LUTS as determined by an IPSS of eight or over, and a $\mathrm{Q}_{\max }$ of less than $15 \mathrm{~mL} / \mathrm{second}$, as measured by non-invasive uroflowmetry, or invasive pressure flow studies, or both (Dunphy 2015; EAU 2017; McNicholas 2016; McVary 2011). The age limitation was based on the observation that the prevalence of $\mathrm{BPH}$ increases in middle-aged and older men, and is infrequent in younger men (Barry 1997; Egan 2016; EAU 2017).

We planned to exclude trials of men with active urinary tract infection; bacterial prostatitis; chronic renal failure; untreated bladder calculi or large diverticula; prostate cancer; urethral stricture disease; and prior prostate, bladder neck, or urethral surgery. We also planned to exclude studies of men with other conditions that affect urinary symptoms, such as neurogenic bladder due to spinal cord injury, multiple sclerosis, or central nervous system disease.

\section{Types of interventions}

We compared the experimental and comparator interventions for the following outcomes. Concomitant interventions had to be the same in the experimental and comparator groups to establish fair comparisons.

\section{Experimental interventions}

- PUL.

\section{Comparator interventions}

- Sham control (or no intervention).

- TURP (monopolar or bipolar).

- Laser ablations of the prostate (e.g. photoselective vaporization of the prostate).

- Laser enucleations of the prostate (e.g. holmium laser enucleations of the prostate).

- Other minimally invasive therapies (e.g. transurethral incision of the prostate, transurethral thermal ablation of the prostate (needle ablation, microwave therapy, and radiofrequency ablative techniques), prostate stent, and prostatic arterial embolization).

- Simple prostatectomy (e.g. open, laparoscopic, and roboticassisted prostatectomy). 


\section{Comparisons}

- PUL versus sham control (or no intervention).

- PUL versus TURP.

- PUL versus laser ablations of the prostate.

- PUL versus laser enucleations of the prostate.

- PUL versus other minimally invasive therapies.

- PUL versus simple prostatectomy.

\section{Types of outcome measures}

We did not use the measurement of the outcomes assessed in this review as an eligibility criterion.

\section{Primary outcomes}

- Urological symptom scores.

- Quality of life.

- Major adverse events.

\section{Secondary outcomes}

- Retreatment.

- Erectile function.

- Ejaculatory function.

- Minor adverse events.

- Acute urinary retention.

- Indwelling urinary catheter.

- Hospital stay.

\section{Method and timing of outcome measurement}

We considered clinically important differences for all outcomes as the basis for rating the certainty of the evidence for imprecision in the 'Summary of findings' tables (Jaeschke 1989; Johnston 2013).

\section{Urological symptom scores}

- Mean change measured as IPSS (also known as American Urological Association Symptom Index).

- We considered an improvement of the IPSS score of three points as the minimal clinically important difference (MCID) to assess efficacy and comparative effectiveness (Barry 1995).

\section{Quality of life}

- Mean change measured as IPSS-quality of life.

- No formal threshold was established for the IPSS-quality of life. We used an MCID of 0.5 to assess efficacy and comparative effectiveness (Brasure 2016; Rees 2015).

\section{Major adverse events}

- For example, postoperative haemorrhage requiring admission or intervention.

- We used the Clavien-Dindo classification system to assess surgical complications (Dindo 2004), and categorized grade III, IV, and V complications as major (Gratzke 2017). We judged the adverse events by severity using the available information described in Roehrborn 2013.

- Based on Guyatt 2011a, we considered a 25\% relative change as threshold for a clinically important difference.
Retreatment

- Events requiring other surgical treatment modalities (e.g. TURP) after intervention.

- Based on Guyatt 2011a, we considered a 25\% relative change as threshold for a clinically important difference.

\section{Erectile function}

- Mean change, measured as total score of International Index of Erectile Function (IIEF)-5 questionnaire (also known as Sexual Health Inventory for Men) (Rosen 1997).

- We considered a difference in IIEF-5 over five points as MCID (Spaliviero 2010).

\section{Ejaculatory function}

- Mean change, measured as Male Sexual Health Questionnaire for Ejaculatory Dysfunction (MSHQ-EjD); (Rosen 2007).

- We used an MCID of 25\% improvement from baseline in MSHQEjD for ejaculatory function (Nickel 2015)

\section{Minor adverse events}

- For example, postoperative fever or pain requiring medication.

- We used the Clavien-Dindo classification system to assess surgical complications (Dindo 2004), and categorized grade I and Il complications as minor (Gratzke 2017). For one of the included studies (Roehrborn 2013), we graded the adverse events as described above.

- Based on Guyatt 2011a, we considered a 25\% relative change as threshold for a clinically important difference.

\section{Acute urinary retention}

- Events requiring catheterization after intervention.

- Based on Guyatt 2011a, we considered a 25\% relative change as threshold for a clinically important difference.

\section{Indwelling urinary catheter}

- Proportion of participants with indwelling catheter at postoperative 24 hours.

- Based on Guyatt 2011a, we considered a 25\% relative change as threshold for a clinically important difference.

\section{Hospital stay}

- Measured in days from admission to discharge.

- We considered a one-day difference in mean hospital stay as the threshold for a clinically important difference.

We considered outcomes measured up to and including 12 months after randomization as short-term and later than 12 months as long-term for urological symptom scores, quality of life, major adverse events, retreatment, erectile function, ejaculatory function, minor adverse events, and acute urinary retention. We assessed indwelling urinary catheter and hospital stay as shortterm only.

\section{Main outcomes for 'Summary of findings' tables}

We presented 'Summary of findings' tables reporting the following outcomes listed according to priority.

- Urological symptom scores.

- Quality of life. 
- Major adverse events.

- Retreatment.

- Erectile function.

- Ejaculatory function.

\section{Search methods for identification of studies}

We performed a comprehensive search with no restrictions on the language of publication or publication status.

\section{Electronic searches}

We initially searched the following sources from inception of each database to 26 May 2017. The date of last search of all databases was 31 January 2019 (Appendix 1).

- The Cochrane Library via Wiley (from 1991).

- Cochrane Database of Systematic Reviews.

- Cochrane Central Register of Controlled Trials.

- Database of Abstracts of Reviews of Effects.

- Health Technology Assessment Database.

- MEDLINE via Ovid (from 1946).

- Embase via Elsevier (from 1947).

- LILACS (Latin American and the Caribbean Health Sciences Literature; www.bireme.br/; from 1982).

- Scopus (from 1966).

- Web of Science (from 1900).

- Google Scholar

We also searched the following.

- ClinicalTrials.gov (www.clinicaltrials.gov/).

- World Health Organization (WHO) International Clinical Trials Registry Platform search portal (apps.who.int/trialsearch/).

- Grey literature repository from the current Grey Literature Report (www.greylit.org/).

\section{Searching other resources}

We tried to identify other potentially eligible trials or ancillary publications by searching the reference lists of retrieved included trials, reviews, meta-analyses, and health technology assessment reports. We also contacted study authors of included trials to identify any further studies that we may have missed. We contacted drug/device manufacturers for ongoing or unpublished trials. We searched abstract proceedings of relevant meetings of the American Urological Association, European Association of Urology, and International Continence Society for 2015 to 2017 from initial date of search and then updated the search for the most recent year (2018) for unpublished studies.

\section{Data collection and analysis}

\section{Selection of studies}

We used EndNote 2016 reference management software to identify and remove potential duplicate records. Two review authors ( $\mathrm{JHJ}$, $\mathrm{BR}$, or KAM) independently scanned the abstracts, titles, or both to determine which studies should be assessed further using Covidence 2013 software. Two review authors (JHJ, BR, or KAM) investigated all potentially relevant records as full text, mapped records to studies, and classified studies as included studies, excluded studies, studies awaiting classification, or ongoing studies in accordance with the criteria for each provided in the Cochrane Handbook for Systematic Reviews of Interventions (Higgins 2011a). We resolved any discrepancies through consensus or recourse to a third review author (PD). We documented reasons for exclusion the Characteristics of excluded studies table. We presented an adapted PRISMA flow diagram showing the process of study selection (Liberati 2009).

\section{Data extraction and management}

We developed a dedicated data abstraction form that we pilot tested ahead of time.

For studies that fulfilled inclusion criteria, two review authors ( $\mathrm{JHJ}$ and BR) independently abstracted the following information, which we provided in the Characteristics of included studies table.

- Study design.

- Study dates.

- Study settings and country.

- Participant inclusion and exclusion criteria (e.g. age, baseline IPSS).

- Participant details, baseline demographics (e.g. age, prostate size, IPSS).

- Number of participants by study and by study arm.

- Details of relevant experimental intervention, such as delivery devices (e.g. size of cystoscope and needle to delivery implants) for PUL and comparator intervention (e.g. monopolar versus bipolar energy, type of laser).

- Definitions of relevant outcomes, and method (e.g. type of instrument, such as IPSS) and timing of outcome measurement (e.g. in months) as well as any relevant subgroups (e.g. based on age, prostate volume, severity of LUTS).

- Study funding sources.

- Declarations of interest by primary investigators.

We extracted outcome data relevant to this Cochrane Review as needed for calculation of summary statistics and measures of variance. For dichotomous outcomes, we obtained numbers of events and totals for population in a $2 \times 2$ table, as well as summary statistics with corresponding measures of variance. For continuous outcomes, we obtained means and standard deviations or data necessary to calculate this information.

We resolved any disagreements by discussion, or, if required, by consultation with a third review author (PD).

We provided information, including trial identifier, about potentially relevant studies in the Characteristics of studies awaiting classification or Characteristics of ongoing studies tables.

We contacted authors of included studies to obtain key missing data as needed.

\section{Dealing with duplicate and companion publications}

In the event of duplicate publications, companion documents, or multiple reports of a primary study, we maximized yield of information by mapping all publications to unique studies and collating all available data. We used the most complete data set aggregated across all known publications. In case of doubt, we gave priority to the publication reporting the longest follow-up associated with our primary or secondary outcomes. 


\section{Assessment of risk of bias in included studies}

Two review authors ( $\mathrm{JHJ}$ and $\mathrm{BR}$ ) independently assessed the risk of bias of each included study. We resolved disagreements by consensus, or by consultation with a third review author (PD). We present a 'Risk of bias' summary figure to illustrate these findings. We further summarized the risk of bias across domains for each outcome in each included study, as well as across studies and domains for each outcome in accordance with the approach for summary assessments of the risk of bias presented in the Cochrane Handbook for Systematic Reviews of Interventions (Higgins 2011b; Sterne 2016).

\section{Assessment of risk of bias in randomized controlled trials}

We assessed risk of bias using Cochrane's 'Risk of bias' assessment tool (Higgins 2011b). We assessed the following domains.

- Random sequence generation (selection bias).

- Allocation concealment (selection bias).

- Blinding of participants and personnel (performance bias).

- Blinding of outcome assessment (detection bias).

- Incomplete outcome data (attrition bias).

- Selective reporting (reporting bias).

- Other sources of bias.

We judged risk of bias domains as 'low risk', 'high risk', or 'unclear risk' and evaluated individual bias items as described in the Cochrane Handbook for Systematic Reviews of Interventions (Higgins 2011b).

For selection bias (random sequence generation and allocation concealment), we evaluated risk of bias at a trial level.

For performance bias (blinding of participants and personnel), we considered all outcomes similarly susceptible to performance bias.

For detection bias (blinding of outcome assessment), we grouped outcomes as susceptible to detection bias (subjective) or not susceptible to detection bias (objective) outcomes.

We defined the following endpoints as subjective outcomes.

- Urological symptom scores.

- Quality of life.

- Major adverse events.

- Erectile function.

- Ejaculatory function.

- Minor adverse events.

We defined the following endpoints as objective outcomes.

- Retreatment.

- Acute urinary retention.

- Indwelling urinary catheter.

- Hospital stay.

We assessed attrition bias (incomplete outcome data) on an outcome-specific basis, and presented the judgement for each outcome separately when reporting our findings in the 'Risk of bias' tables.
For reporting bias (selective reporting), we evaluated risk of bias at a trial level.

\section{Assessment of risk of bias in non-randomized controlled trials}

We planned to assess risk of bias in non-RCTs with ROBINS-I: a tool for assessing risk of bias in non-randomized studies of interventions (Sterne 2016).

- Bias due to confounding

- Bias in selection of participants into the study.

- Bias in classification of interventions.

- Bias due to deviations from intended interventions.

- Bias due to missing data.

- Bias in measurement of outcomes.

- Bias in selection of the reported result.

We planned to judge risk of bias domains as 'low risk', 'moderate risk', 'serious risk', 'critical risk', or 'no information' and evaluate individual bias items as described in Sterne 2016.

\section{Measures of treatment effect}

We expressed dichotomous data as risk ratios (RRs) with 95\% confidence interval (Cls). We expressed continuous data as mean differences (MDs) with 95\% Cls.

\section{Unit of analysis issues}

The unit of analysis was the individual participant. For cluster-trials, or trials with more than two intervention groups, we planned to incorporate these study designs in meta-analysis in accordance with guidance provided in the Cochrane Handbook for Systematic Reviews of Interventions (Higgins 2011c).

\section{Dealing with missing data}

We obtained missing data from study authors and performed intention-to-treat analyses if data were available. We investigated attrition rates (e.g. dropouts, losses to follow-up, and withdrawals), and critically appraised issues of missing data. We did not impute missing data.

\section{Assessment of heterogeneity}

We planned to identify heterogeneity (inconsistency) through visual inspection of the forest plots to assess the amount of overlap of $\mathrm{Cls}$, and the $\mathrm{I}^{2}$ statistic, which quantified inconsistency across studies to assess the impact of heterogeneity on the meta-analysis (Higgins 2002; Higgins 2003). We planned to interpret the $\mathrm{I}^{2}$ statistic as follows (Deeks 2011).

- $0 \%$ to $40 \%$ : may not be important.

- $30 \%$ to $60 \%$ : may indicate moderate heterogeneity.

- $50 \%$ to $90 \%$ : may indicate substantial heterogeneity.

- $75 \%$ to $100 \%$ : considerable heterogeneity.

\section{Assessment of reporting biases}

We tried to obtain study protocols to assess for selective outcome reporting.

As we included only one study for each comparison, we could not use funnel plots to assess small-study effects. 


\section{Data synthesis}

Given that we included only one study for each comparison, we only reported single study data using Review Manager 5 software (Review Manager 2014).

\section{Subgroup analysis and investigation of heterogeneity}

We expected the following characteristics to introduce clinical heterogeneity, and planned to carry out subgroup analyses with investigation of interactions.

- Patient age (less than 65 years versus 65 years and older).

- Prostate volume (40 mL or less versus greater than $40 \mathrm{~mL}$ ).

- Severity of LUTS based on IPSS (score 19 (moderately symptomatic) or less versus greater than 19 (severely symptomatic)).

These subgroup analyses are based on the following observations.

- Age is a well-known risk factor of BPH surgery. Older people have a higher rate of postoperative complications compared with younger people (Bhojani 2014; Pariser 2015). The age cutoff was based on the WHO definition of old age (WHO 2002).

- The outcomes and complications of minimally invasive procedures, such as TURP correlate with prostate volume (Reich 2008). The prostate volume cut-off greater than $40 \mathrm{~mL}$ was based on this being the most commonly used threshold to distinguish 'small' from 'large' for the indication of treatment with a 5-alpha reductase inhibitor (EAU 2017).

- The relationship between changes in IPSS scores and patient global ratings of improvement is influenced by the baseline scores (Barry 1995).

We planned to perform subgroup analyses limited to the primary outcomes.

\section{Sensitivity analysis}

We planned to perform sensitivity analyses limited to the primary outcomes to explore the influence of the following factor (when applicable) on effect sizes.

- Restricting the analysis in RCTs by taking into account risk of bias, by excluding studies at 'high risk' or 'unclear risk'.

\section{'Summary of findings' tables}

We presented the overall certainty of the evidence for each outcome according to the GRADE approach (Guyatt 2008). For each comparison, two review authors ( $\mathrm{JHJ}$ and $\mathrm{BR}$ ) independently rated the certainty of evidence for each outcome as 'high', 'moderate', 'low', or 'very low' using GRADEpro GDT 2015. We resolved any discrepancies by consensus, or, if needed, by arbitration by a third review author (PD). For each comparison, we presented a summary of the evidence for the main outcomes in a 'Summary of findings' table, which provides key information about the best estimate of the magnitude of the effect in relative terms and absolute differences for each relevant comparison of alternative management strategies; numbers of participants and studies addressing each important outcome; and the rating of the overall confidence in effect estimates for each outcome (Guyatt 2011b; Schünemann 2011a).

For RCTs, we took into account five criteria related to internal validity (risk of bias, inconsistency, imprecision, and publication bias), and external validity (such as directness) of results for downgrading the certainty of evidence for a specific outcome (Schünemann 2011b). For non-RCTs, we planned to take into account three criteria for upgrading the certainty of evidence (large magnitude of effects, all plausible confounding that would reduce a demonstrated effect or suggest a spurious effect when results show no effect, and dose-response gradient) (Schünemann 2011b).

\section{RES U L T S}

\section{Description of studies}

\section{Results of the search}

We identified 657 records through electronic database searching, including 10 records in trials registers. We found no records in the grey literature repository, and reference lists of retrieved included trials and reviews. After removal of duplicates, we screened the titles and abstracts of 283 records, and excluded 252 records. We screened 31 full-text articles and excluded one record due to wrong study design. We found one study awaiting classification (two records). We included two studies ( 28 records) in the review. The flow of literature through the assessment process is shown in the PRISMA flowchart (Figure 1). 
Figure 1. PRISMA flow diagram.

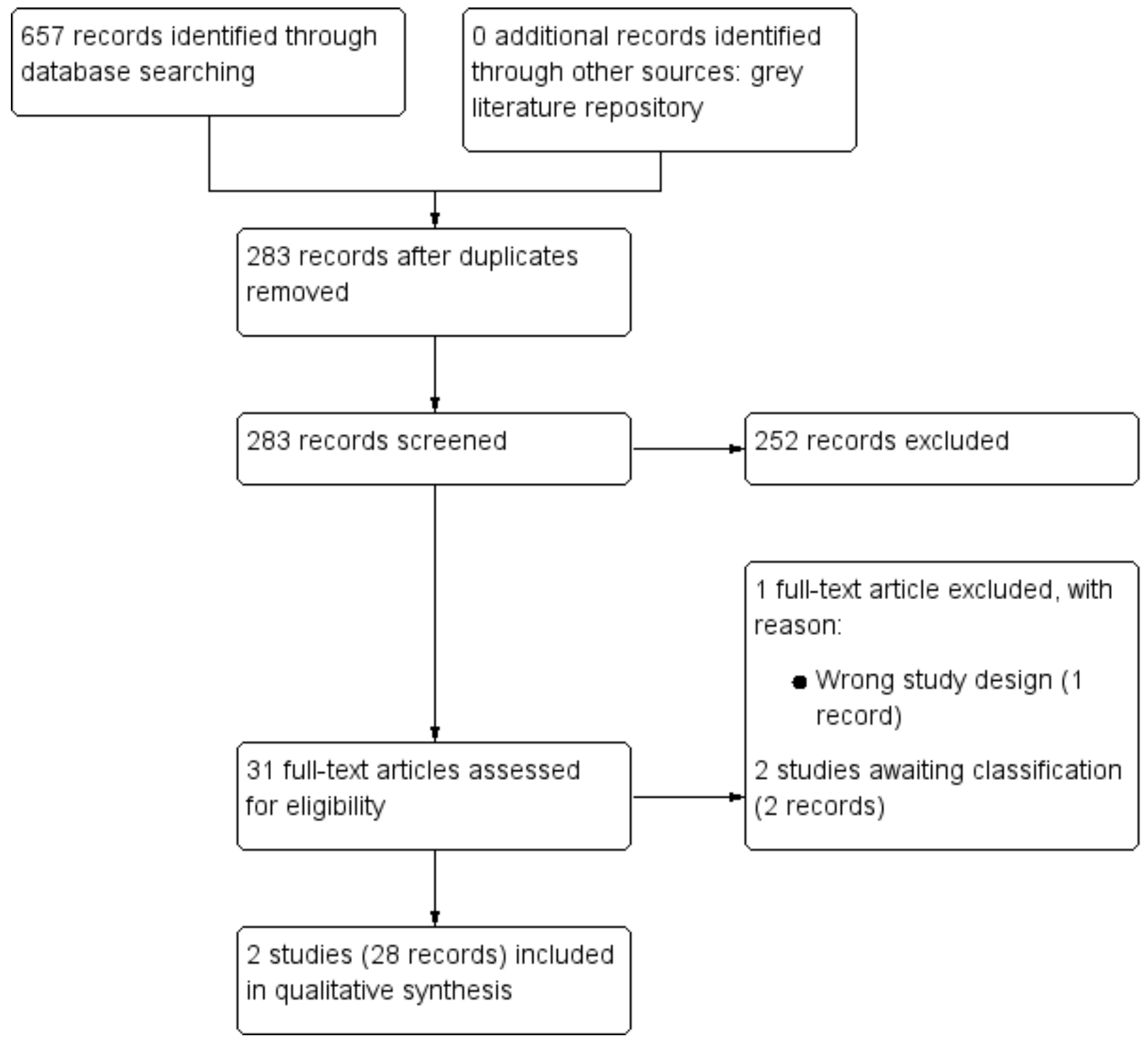

\section{Included studies}

Details of included studies are presented elsewhere (Characteristics of included studies; Table 1; Table 2). In brief,

- Roehrborn 2013 randomized 206 men of 50 years of age or older with an IPSS greater than $12, \mathrm{Q}_{\max } 12 \mathrm{~mL} /$ second or less, and prostate volume of $30 \mathrm{~cm}^{3}$ to $80 \mathrm{~cm}^{3}$ to PUL versus sham surgery.

- Gratzke 2017 randomized 91 men of 50 years of age or older with an IPSS greater than $12, \mathrm{Q}_{\max } 15 \mathrm{~mL} /$ second or less, and prostate volume $60 \mathrm{~cm}^{3}$ or less to PUL versus TURP.

\section{Source of data}

We identified the two published full-text studies through our electronic database search (Gratzke 2017; Roehrborn 2013). Both trials were published in English. We attempted to contact the corresponding authors of the included trials to obtain additional information on study methodology and results, and received reply from Roehrborn 2013 (Appendix 2).

\section{Study design and settings}

Both trials were multicentre, parallel, RCTs across Europe, US, Canada, and Australia. One study was reported as "open-label" in the protocol (Gratzke 2017), but the other was unclear for the blinding (single blinded in protocol, but double blinded in full-text publication (Roehrborn 2013). The studies were performed from 2011 to 2013.

\section{Participants}

We included 297 randomized participants (PUL 185, sham 66, TURP 46). Out of 297 participants, 275 were included in the efficacy (urological symptom scores: PUL 177, sham 66, TURP 32) and 285 were included in safety (adverse events: PUL 185, sham 66, TURP 35 ) analysis. The mean age was 65.6 years, mean IPSS was 22.7 , and mean $\mathrm{Q}_{\max }$ was $8.9 \mathrm{~mL} /$ second. Mean prostate volume was $42.2 \mathrm{~mL}$. 
Both studies included participants with LUTS of a total IPSS more than 12 and prostate volume less than $80 \mathrm{~mL}$ (Gratzke 2017: less than $60 \mathrm{~mL}$, Roehrborn 2013: $30 \mathrm{~mL}$ to $80 \mathrm{~mL}$ ). One study used a $\mathrm{Q}_{\max }$ of $15 \mathrm{~mL} / \mathrm{second}$ or less (Gratzke 2017) and one study used $15 \mathrm{~mL} /$ second (Roehrborn 2013) as an inclusion criterion. Major exclusion criteria included active urinary tract infection, urinary retention, raised PSA level suspicious of prostate cancer, history of prior prostate-related surgery such as TURP or laser procedure, and other medical conditions or medical comorbidities that represented relative or absolute contraindications for TURP or PUL.

\section{Intervention(s) and comparator(s)}

Both studies used permanent intraprostatic UroLift implants (NeoTract, Inc., Pleasanton, CA) as interventions. Roehrborn 2013 used sham surgery and Gratzke 2017 used TURP as a comparator. After rigid cystoscopy was performed, the implant delivery device was inserted into the cystoscopic sheath. Under cystoscopic visualization, PUL implants were permanently implanted to retract obstructing lateral lobes and widen the urethral lumen (Gratzke 2017; McNicholas 2016; Roehrborn 2013). For sham procedures, after a rigid cystoscopy was performed, the surgeon requested the devices which were opened but not deployed. A device was not inserted, but was deployed four times outside the patients' body to simulate the device sounds (Roehrborn 2013). TURP procedures were performed in accordance with normal standards and practices of participating centres with no other details being provided about the devices being used by Gratzke 2017. Roehrborn 2013 conducted almost all PUL procedures under local anaesthesia. Gratzke 2017 appeared to have performed PUL procedures under general or spinal anaesthesia.

While Roehrborn 2013 reported five-year follow-up data for the PUL group as an extended open-label study, we, in accordance with our published protocol, only used three-month follow-up data for which there was a concurrent comparison groups (PUL and sham). The follow-up duration of Gratzke 2017 was 24 months.

\section{Comparisons}

The included two studies compared PUL to sham (Roehrborn 2013) or TURP (Gratzke 2017). We found no studies comparing PUL to laser ablations of the prostate, laser enucleations of the prostate, other minimally invasive therapies, or simple prostatectomy.

\section{Outcomes}

We identified reporting of all primary outcomes in each of the included studies for both comparisons. In addition, all secondary outcomes were reported in each of the included studies except for indwelling catheter requirements.

\section{Funding sources and conflicts of interest}

Both included studies were supported by the same medical device company and also reported the authors having financial relationships with the same as well as other pharmaceutical or medical device companies, or both.

\section{Excluded studies}

We excluded one abstract that was a single armed and posthoc analysis of the included study (Gratzke 2018). See Characteristics of excluded studies table.

\section{Studies awaiting classification and ongoing trials}

We found one study awaiting classification that appeared to meet inclusion criteria. Details of this study are presented in the Characteristics of studies awaiting classification table. In brief, this is a non-randomized prospective study comparing a men with similar symptoms of LUTS undergoing either PUL or prostatic arterial embolization. The study has yet to begin accrual at the time of completion of this review. We plan to include its findings in future updates.

\section{Risk of bias in included studies}

See Figure 2; Figure 3. 
Figure 2. Risk of bias graph: review authors' judgements about each risk of bias item presented as percentages across all included studies.

\begin{tabular}{|r|} 
Random sequence generation (selection bias) \\
Allocation concealment (selection bias) \\
Blinding of outcome assessment (detection bias): Subjective outcomes \\
Blinding of outcome assessment (detection bias): Objective outcomes \\
Incomplete outcome data (attrition bias): Urological symptom scores/QoL \\
Incomplete outcome data (attrition bias): Major adverse events/minor adverse events \\
Incomplete outcome data (attrition bias): Retreatment \\
Incomplete outcome data (attrition bias): Erectile function \\
Incomplete outcome data (attrition bias): Ejaculatory function \\
Incomplete outcome data (attrition bias): Acute urinary retention \\
Incomplete outcome data (attrition bias): Indwelling catheter \\
Incomplete outcome data (attrition bias): Hospital stay \\
Selective reporting (reporting bias)
\end{tabular}


Figure 3. Risk of bias summary: review authors' judgements about each risk of bias item for each included study.

\begin{tabular}{|c|c|c|c|c|c|c|c|c|c|c|c|c|c|c|c|}
\hline & 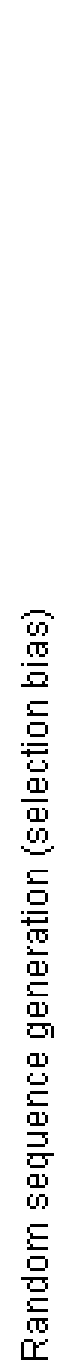 & 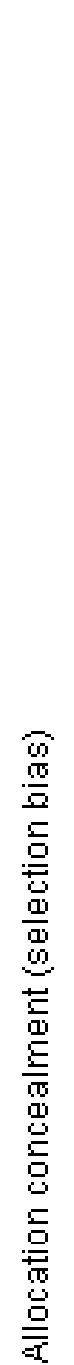 &  &  &  & 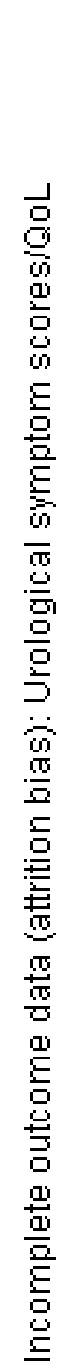 & 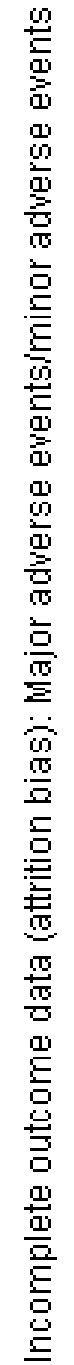 & 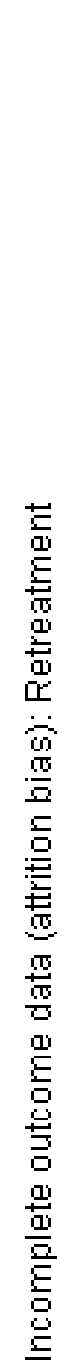 & 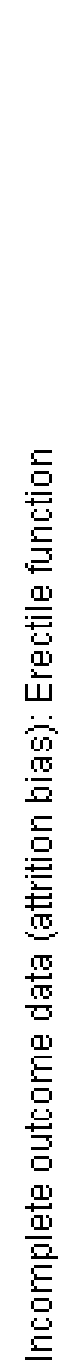 & 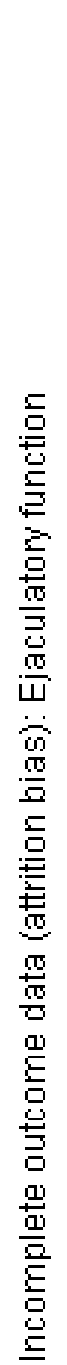 & 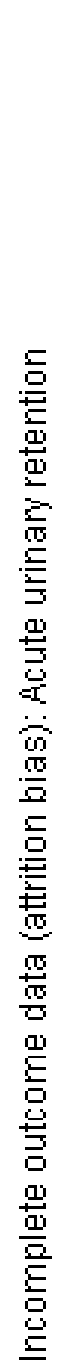 & 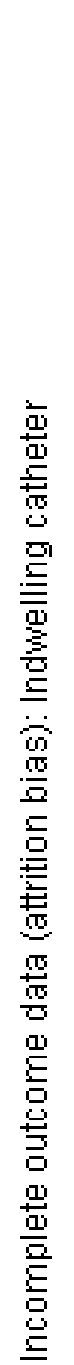 & 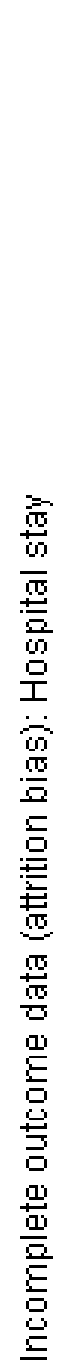 & 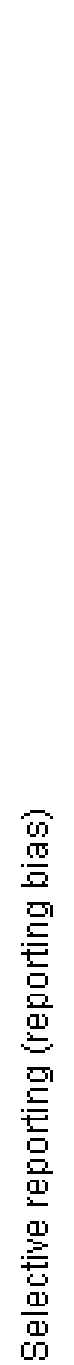 & 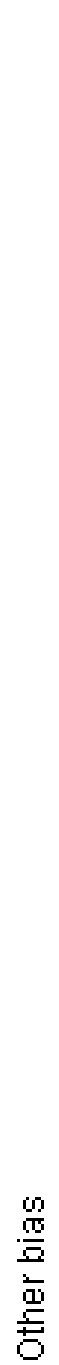 \\
\hline Gratzke 2017 & + & + & & & + & $?$ & + & + & & & & $?$ & & + & + \\
\hline Roehrborn 2013 & + & + & & + & + & + & + & + & + & & + & $?$ & + & + & + \\
\hline
\end{tabular}

\section{Allocation}

\section{Random sequence generation}

We rated both studies at low risk of bias since they used appropriate methods of random sequence generation.

\section{Allocation concealment}

We rated both studies at low risk of bias since both indicated appropriate concealment of participant allocation.

\section{Blinding}

Roehrborn 2013 reported that 'double blinding' was maintained for three months after intervention, but there are concerns whether this was the case given that the study protocol referred to a single-blinded design. However, the author clarified blinding of participants and outcome assessor.

\section{Blinding of participants and personnel}

We rated both studies at high risk of bias due to a lack of blinding (Gratzke 2017: non-blinded; Roehrborn 2013: non-blinded for personnel).

\section{Blinding of outcome assessment}

- Subjective outcomes (urological symptom scores, quality of life, major adverse events, erectile function, ejaculatory function, and minor adverse events): we rated one study at low risk of bias based on author's response (Roehrborn 2013), but we judged the other at high risk of bias (Gratzke 2017). 
- Objective outcomes (retreatment, acute urinary retention, indwelling urinary catheter, and hospital stay): we rated both studies at low risk of bias for these outcomes because these outcomes are unlikely to be affected by lack of blinding.

\section{Incomplete outcome data}

While Roehrborn 2013 only reported short-term outcomes (up to 12 months' follow-up), Gratzke 2017 reported both short- and long-term (longer than 12 months' follow-up) outcome data. We rated the risk of bias separately for all outcomes in Gratzke 2017 according to the timing of outcome measurement (short term and long term) but these judgements were identical with the exception of major adverse events.

- Urological symptom scores and quality of life: while rating one study at low risk of bias (Roehrborn 2013), we judged the other at unclear risk of bias (Gratzke 2017).

- Major and minor adverse events: we rated both studies at low risk of bias.

- Retreatment: we rated both studies at low risk of bias.

- Erectile function: we rated one study at low risk of bias (Roehrborn 2013), and the other at high risk of bias (Gratzke 2017).

- Ejaculatory function: we rated both studies at high risk of bias.

- Acute urinary retention: we rated both studies at low risk of bias.

- Indwelling urinary catheter: we rated both studies at unclear risk of bias due to lack of information.

- Hospital stay: we rated both studies at low risk of bias.

\section{Selective reporting}

We rated both studies at low risk of bias given the availability of registration protocols and concordance of planned and actual analyses for all of our predefined outcomes.

\section{Other potential sources of bias}

We rated both studies at low risk of bias; no other sources of bias were identified.

\section{Effects of interventions}

See: Summary of findings for the main comparison PUL compared to TURP for the treatment of lower urinary tract symptoms in men with benign prostatic hyperplasia (short term); Summary of findings 2 PUL compared to TURP for the treatment of lower urinary tract symptoms in men with benign prostatic hyperplasia (long term); Summary of findings 3 PUL compared to sham for the treatment of lower urinary tract symptoms in men with benign prostatic hyperplasia (short term)

\section{Prostatic urethral lift versus sham (short term)}

We found one study comparing PUL versus sham with short-term follow-up which randomized 206 participants (PUL 140, sham 66) (Roehrborn 2013). All randomized participants were included in the analysis for primary and secondary outcomes except for the outcomes of erectile function (197 participants; PUL 132, sham 65), ejaculatory function (144 participants; PUL 94, sham 50), and hospital stay (201 participants; PUL 137, sham 64).

There were no studies with long-term follow-up. The results are presented in a summary of findings table (Summary of findings 3 ).

\section{Primary outcomes}

\section{Urological symptom scores}

PUL may lead to a clinically important reduction in urological symptom scores (MD $-5.20,95 \% \mathrm{Cl}-7.44$ to -2.96$)$. We rated the certainty of the evidence as low, downgrading for study limitations and imprecision (Analysis 1.1).

\section{Quality of life}

PUL likely results in similar improvement in quality of life (MD -1.20, $95 \% \mathrm{Cl}-1.67$ to -0.73 ) as sham surgery. We rated the certainty of the evidence as moderate, downgrading for study limitations (Analysis 1.2).

\section{Major adverse events}

We are very uncertain about the effects of PUL on major adverse events (RR $3.30,95 \% \mathrm{Cl} 0.41$ to 26.28 ). We rated the certainty of the evidence as very low, downgrading for study limitations and very serious imprecision (Analysis 1.3).

\section{Secondary outcomes}

\section{Retreatment}

There were no retreatments in either study group at three months' follow-up. We rated the certainty of the evidence as low, downgrading for study limitations and imprecision.

\section{Erectile function}

PUL likely results in little to no difference in erectile function (MD $1.40,95 \% \mathrm{Cl}-3.24$ to 0.44 ). We rated the certainty of the evidence as moderate, downgrading for study limitations (Analysis 1.5).

\section{Ejaculatory function}

PUL likely results in little to no difference in ejaculatory function (MD $0.50,95 \% \mathrm{Cl}-0.38$ to 1.38 ). We rated the certainty of the evidence as moderate, downgrading for study limitations (Analysis 1.4).

\section{Minor adverse events}

PUL likely increases minor adverse events (RR 1.69, 95\% Cl 1.33 to 2.16). PUL would result in 355 more minor adverse events per 1000 men ( $95 \% \mathrm{Cl} 170$ more to 598 more). We rated the certainty of the evidence as moderate, downgrading for study limitations (Analysis 1.6).

\section{Acute urinary retention}

We are very uncertain about the effects of PUL on acute urinary retention ( $\mathrm{RR} 0.47,95 \% \mathrm{Cl} 0.03$ to 7.42 ). We rated the certainty of the evidence as very low, downgrading for study limitations and very serious imprecision (Analysis 1.7).

\section{Indwelling urinary catheter}

The study did not report the need for an indwelling urinary catheter.

\section{Hospital stay}

PUL likely results in little to no difference in hospital stay (MD 0.03 , $95 \% \mathrm{Cl}-0.09$ to 0.15 ). We rated the certainty of the evidence as moderate, downgrading for study limitations (Analysis 1.8). 


\section{Subgroup and sensitivity analysis}

We were unable to perform any predefined secondary analyses because there were no relevant data.

\section{Prostatic urethral lift versus transurethral resection of prostate (short term)}

One trial compared PUL versus TURP (Gratzke 2017). We included 72 participants (PUL 40, TURP 32) in the analysis for urological symptom scores and quality of life; 79 participants (PUL 44, TURP 35 ) in the analysis for major adverse events, retreatment, minor adverse events, acute urinary retention, indwelling urinary catheter, and hospital stay; and 59 participants (PUL 32, TURP 27) in the analysis for erectile and ejaculatory function. The results are presented in a summary of findings table (Summary of findings for the main comparison).

\section{Primary outcomes}

\section{Urological symptom scores}

PUL may result in a substantially lesser improvement in urological symptom scores compared with TURP (MD 4.50, 95\% Cl 1.10 to 7.90 ; low-certainty evidence). We rated the certainty of the evidence as low, downgrading for study limitations and imprecision (Analysis 2.1).

\section{Quality of life}

PUL may result in little to no difference in quality of life (MD 0.30 , $95 \% \mathrm{Cl}-0.49$ to 1.09 ) compared to TURP. We rated the certainty of the evidence as low, downgrading for study limitations and imprecision (Analysis 2.2).

\section{Major adverse events}

We are very uncertain about the effects of PUL on major adverse events (RR $0.64,95 \% \mathrm{Cl} 0.18$ to 2.19 ). We rated the certainty of the evidence as very low, downgrading for study limitations and very serious imprecision (Analysis 2.3).

\section{Secondary outcomes}

\section{Retreatment}

We are very uncertain about the effects of PUL on retreatment (RR $1.19,95 \% \mathrm{Cl} 0.21$ to 6.75 ). We rated the certainty of the evidence as very low, downgrading for study limitations and very serious imprecision (Analysis 2.4).

\section{Erectile function}

PUL likely results in little to no difference in erectile function (MD $0.80,95 \% \mathrm{Cl}-1.50$ to 3.10 ). We rated the certainty of the evidence as moderate, downgrading for study limitations (Analysis 2.5).

\section{Ejaculatory function}

PUL likely preserves ejaculatory function to a similar extent as TURP (MD 5.00, 95\% Cl 3.08 to 6.92). We rated the certainty of the evidence as moderate, downgrading for study limitations (Analysis 2.6).

\section{Minor adverse events}

PUL may result in slightly fewer minor adverse events compared with TURP (RR $0.88,95 \% \mathrm{Cl} 0.70$ to 1.09 ). PUL would result in 103 fewer minor adverse events per 1000 men $(95 \% \mathrm{Cl} 257$ fewer to 77 more). We rated the certainty of the evidence as low, downgrading for study limitations and imprecision (Analysis 2.7).

\section{Acute urinary retention}

We are very uncertain about the effects of PUL on acute urinary retention, which may be much higher or lower (RR $7.20,95 \% \mathrm{Cl}$ 0.40 to 129.38 ). We rated the certainty of the evidence as very low, downgrading for study limitations and very serious imprecision (Analysis 2.8).

\section{Indwelling urinary catheter}

PUL may reduce the need for an indwelling urinary catheter (RR $0.46,95 \% \mathrm{Cl} 0.25$ to 0.84 ). PUL would result in 293 fewer indwelling urinary catheters per 1000 men ( $95 \% \mathrm{Cl} 87$ fewer to 407 fewer). We rated the certainty of the evidence as low, downgrading for study limitations and imprecision (Analysis 2.9).

\section{Hospital stay}

PUL may result in a small reduction in hospital stay, but this may not represent a clinically important difference (MD $-0.90,95 \% \mathrm{Cl}$ -1.32 to -0.48 ). We rated the certainty of the evidence as low, downgrading for study limitations and imprecision (Analysis 2.10).

\section{Subgroup and sensitivity analysis}

We were unable to perform secondary analyses because there were no relevant data or too few data in the included studies.

\section{Prostatic urethral lift versus transurethral resection of prostate (long term)}

We included one trial for long-term follow-up of PUL versus TURP (Gratzke 2017). We included 69 participants (PUL 37, TURP 32) in the analysis for urological symptom scores and quality of life; 79 participants (PUL 44, TURP 35) in the analysis for retreatment; 57 participants in the analysis for erectile (PUL 29, TURP 28); 56 participants in the analysis for ejaculatory function (PUL 29, TURP 27); and 80 participants (PUL 45, TURP 35) in the analysis for minor adverse events. The results are presented in a summary of findings table (Summary of findings 2).

\section{Primary outcomes}

\section{Urological symptom scores}

PUL may reduce urological symptom scores less than TURP (MD $6.10,95 \% \mathrm{Cl} 2.16$ to 10.04 ). We rated the certainty of the evidence as low, downgrading for study limitations and imprecision (Analysis 3.1).

\section{Quality of life}

PUL may result in little to no difference in quality of life (MD 0.80 , $95 \% \mathrm{Cl} 0.00$ to 1.60 ). We rated the certainty of the evidence as low, downgrading for study limitations and imprecision (Analysis 3.2).

\section{Major adverse events}

The study did not report major adverse events.

\section{Secondary outcomes}

\section{Retreatment}

We are very uncertain about the effects of PUL on retreatment (RR $2.39,95 \% \mathrm{Cl} 0.51$ to 11.10$)$. We rated the certainty of the evidence 
as very low, downgrading for study limitations and very serious imprecision (Analysis 3.3).

\section{Erectile function}

PUL likely results in little to no difference in erectile function (MD $1.60,95 \% \mathrm{Cl}-0.80$ to 4.00 ). We rated the certainty of the evidence as moderate, downgrading for study limitations (Analysis 3.4).

\section{Ejaculatory function}

PUL may preserve ejaculatory function to a greater extent than TURP (MD 4.30, 95\% Cl 2.17 to 6.43 ). We rated the certainty of the evidence as low, downgrading for study limitations and imprecision (Analysis 3.5).

\section{Minor adverse events}

PUL may result in slightly fewer minor adverse events (RR 0.92 $95 \% \mathrm{Cl} 0.72$ to 1.17). PUL would result in 64 fewer minor adverse events per 1000 men ( $95 \% \mathrm{Cl} 224$ fewer to 136 more). We rated the certainty of the evidence as low, downgrading for study limitations and imprecision (Analysis 3.6).

\section{Acute urinary retention}

The study did not report acute urinary retention.

\section{Indwelling urinary catheter}

The study did not report the need for an indwelling urinary catheter.

\section{Hospital stay}

The study did not report hospital stay.

\section{Subgroup and sensitivity analysis}

We were unable to perform secondary analyses because there were no relevant data.

\section{DISCUSSION}

\section{Summary of main results}

We included two studies with 297 randomized participants comparing PUL to sham or TURP.

\section{Prostatic urethral lift versus sham}

Based on one study with comparative data up to three months only, PUL may lead to reduce urological symptoms and likely improves quality of life more than sham surgery. We are very uncertain about the effects of PUL on major adverse events. There were no reported retreatments in either study group. PUL likely results in little to no difference in erectile and ejaculatory function.

\section{Prostatic urethral lift versus transurethral resection of prostate}

Short-term, PUL may result in a substantially lesser reduction of urological symptoms than TURP although quality of life may be similar. We are uncertain about the effects of PUL on major adverse events and retreatment rates. While there may be no difference in erectile function, ejaculatory function may be better preserved.

Long-term, PUL may also result in a substantially lesser reduction of urological symptoms than TURP although quality of life may be similar. We found no evidence on major adverse events. Also long-term, there may be no difference in erectile function, but ejaculatory function may be better preserved.

We were unable to perform any of the predefined secondary analysis for any comparisons.

We were unable to assess any other comparisons.

\section{Overall completeness and applicability of evidence}

As we found only two trials of two comparisons in this review, this body of evidence has important limitations with regards to applicability to contemporary practice.

- Given the principal mechanism of PUL to compress a prostatic adenoma using a series of implants, men with obstructing median lobes may receive limited therapeutic benefit (McNicholas 2016; Walsh 2017). Roehrborn 2013 excluded men with median lobe obstruction. Gratzke 2017 did not describe the presence of median lobe as an exclusion criteria. Both studies included participants with a prostate volume of less than $80 \mathrm{~mL}$. Both issues limit generalizability with regards to men presenting with LUTS secondary to BPH being considered for surgical management.

- While Gratzke 2017 reported the severity of adverse events based on the Clavien-Dindo classification system, Roehrborn 2013 used a different definition of serious adverse events (e.g. stress incontinence and transfusion were considered as serious adverse events without any definitions being provided). Varied definitions and terminology limited comparability and interpretation.

- When comparing PUL versus sham, the study duration was extremely short (three months) thereby limiting the reported assessment.

- In this review, anaesthesia methods by study varied. While most US-based series were performed under local anaesthesia with or without penile block (Perera 2015), European centres used general or spinal anaesthesia in almost all participants (Gratzke 2017). This appears relevant to a subset of outcomes such as length of hospital stay and acute urinary retention.

\section{Quality of the evidence}

We consistently downgraded the certainty of the evidence. The most common reasons for downgrading were study limitations (issues surrounding blinding of participants, personnel, and outcome assessor): given the blinding that is a fundamental safety guard for RCTs, it may result in exaggerated effect size estimates. In addition, we frequently downgraded for imprecision due to wide $\mathrm{Cls}$ that crossed the assumed threshold of clinically important differences, usually in the setting of few events.

For ejaculatory function, we also downgraded for attrition bias due high rates of participants not included in the analysis (Gratzke 2017: 30.0\%, Roehrborn 2013: 30.1\%).

\section{Potential biases in the review process}

Despite a comprehensive search strategy without any publication or language restrictions, we only found two RCTs. While both included studies used the same criteria on our review outcome measurement, the existing paucity of RCTs may be a source of bias to reaching the conclusion. 
The small number of studies included in this review were insufficient to generate funnel plots; therefore, the risk of publication bias may have been underestimated. Both included studies were supported by a device company. Therefore, publication bias and favourable reporting, owing to commercial interests with the current method of PUL, cannot be discounted.

\section{Agreements and disagreements with other studies or reviews}

We found a few published systematic reviews to elucidate the effects of PUL for the treatment of LUTS in men with BPH (Jones 2016; Magistro 2018; Perera 2015; Sanchez-Gomez 2015). While concluding that PUL was well-tolerated, improved urinary symptoms, and stream, while preserving sexual function, they combined the data from RCTs (Gratzke 2017; Roehrborn 2013) and non-RCTs (case series or secondary cross-over designed study of Roehrborn 2013). Limited published reports of varying quality in the meta-analysis cause a high degree of heterogeneity. Therefore, we believe that our review was the most rigorous in its methodology; this included a published protocol, a focus on patient-important outcomes, an exhaustive literature search, and an assessment of the certainty of the evidence using GRADE.

In addition, Roehrborn 2013 reported a cumulative retreatment rate of $10.7 \%$ at three years and $13.6 \%$ at five years for PUL (unblinded single arm follow-up) while Gratzke 2017 reported no difference $(6.8 \%(3 / 44)$ of PUL and $5.7 \%(2 / 35)$ of TURP at 1 year). Based on one systematic review and varying by study, $1.5 \%$ to $16 \%$ of participants underwent TURP after PUL due to insufficient treatment response at the 12-month follow-up (Perera 2015). Meanwhile, one retrospective study of 6430 men undergoing TURP in the US reported the rate of repeated surgical intervention to be only $4 \%$ at three years after surgery (Strope 2015). Based on the included trial evidence, we are uncertain about the effects of PUL on retreatment risk due to study limitations and imprecision.

\section{AUTHORS' CONCLUSIONS}

\section{Implications for practice}

PUL may improve urological symptom scores and quality of life similarly as sham surgery short term. Compared to TURP, it is less effective in improving urological symptom scores both short and long term but may offer advantages with regards to the preservation of ejaculatory function. There is considerable uncertainty or lack of evidence (or both) with regards to the risk of major adverse events and retreatment rates over time.

\section{Implications for research}

We see the following research priorities.

- There appears a critical need for further studies of greater methodological rigor comparing PUL to TURP as well as other treatment modalities such as convective radiofrequency water vapour thermal therapy or holmium laser enucleation. These studies should be of sufficient duration (24 months or longer) and transparently report on treatment-related adverse events and retreatment rates.

- There are few data to help inform which men may be most suitable for PUL based on characteristics such as age, prostate volume, and symptom score. Studies preplanned for subgroup analyses and studies focusing on subsets of men would be helpful.

\section{ACKNOWLEDGEMENTS}

We are very grateful to Tobias Kohler, Philippe Violette, and Sean McAdams for assistance in the preparation of this review. We thank Cochrane Urology and our contact editor Andrew Shepherd for supporting this review. 


\section{R E F E R E N C E S}

\section{References to studies included in this review}

\section{Gratzke 2017 \{published data only\}}

Barber N, Sønsken J, Gratze C, Speakman M, Berges R, Wetterauer $\mathrm{U}$, et al. BPH6 randomized study of prostatic urethral lift (PUL) vs transurethral resection of the prostate (TURP): outcomes and patient satisfaction. Journal of Urology 2015;193(Suppl 4):e19. [DOI: 10.1016/j.juro.2015.02.104]

Chin P, Sønsken J, Barber N, Gratzke C, Speakman M, Berges R, et al. BPH6 trial: a multi-centre, prospective, randomised study of the prostatic urethral lift vs. transurethral resection of the prostate (TURP). BJU International 2015;115(Suppl 4):10. [DOI: 10.1111/bju.13072]

Chin P, Woo H, Speakman M, Sønksen J, Gratzke C. Improved sleep after TURP and prostatic urethral lift (PUL): prospective, randomized study. BJU International 2017;119(Suppl 2):82. [DOI: 10.1111/bju.13752]

* Gratzke C, Barber N, Speakman MJ, Berges R, Wetterauer U, Greene $D$, et al. Prostatic urethral lift vs transurethral resection of the prostate: 2-year results of the $\mathrm{BPH} 6$ prospective, multicentre, randomized study. BJU International 2017;119(5):767-75. [DOI: 10.1111/bju.13714]

Gratzke C, Barber NJ, Speakman MJ, Berges R, Wetterauer U, Greene D, et al. Two year results of the BPH6 trial: a multicenter, prospective, randomized study of the prostatic urethral lift (PUL) vs transurethral resection of the prostate (TURP). European Urology Supplements 2016; Vol. 15, issue 3:e1076-a. [DOI: 10.1016/S1569-9056(16)61077-1]

Gratzke C, Chin P, Barber N, Speakman M, Berges R, Wetterauer U, et al. BPH6 trial two year results: the multinational, prospective, randomised study of the prostatic urethral lift (PUL) compared to transurethral resection of the prostate (TURP). BJU International 2016; Vol. 117, issue Suppl 3:17-8. [DOI: 10.1111/bju.13452]

NCT01533038. BPH-6: comparison of the UroLift System to TURP for benign prostatic hyperplasia. clinicaltrials.gov/ ct2/show/NCT01533038. https://ClinicalTrials.gov/show/ NCT01533038, (first received 15 February 2012). [NCT01533038]

Sønksen J, Barber NJ, Speakman MJ, Berges R, Wetterauer U, Greene D, et al. Prospective, randomized, multinational study of prostatic urethral lift versus transurethral resection of the prostate: 12-month results from the BPH6 study. European Urology 2015; Vol. 68, issue 4:643-52. [DOI: 10.1016/ j.eururo.2015.04.024]

Sønsken J, Barber N, Speakman M, Berges R, Wetterauer U, Greene D, et al. Multi-national, prospective, randomized study of the prostatic urethral lift (PUL) vs. transurethral resection of the prostate (TURP): two year results. Journal of Urology 2016;195(Suppl 4):e456. [DOI: 10.1016/j.juro.2016.02.1468]

\section{Roehrborn 2013 \{published data only\}}

Barkin J. Prospective, randomized, controlled study of fiveyear results on prostatic urethral lift (PUL). Canadian Urological Association Journal 2017;11(9):S326. [DOI: 10.5489/cuaj.4896]

McVary KT, Gange SN, Shore ND, Bolton DM, Cowan BE, Brown BT, et al. Treatment of LUTS secondary to BPH while preserving sexual function: randomized controlled study of prostatic urethral lift. Journal of Sexual Medicine 2014; Vol. 11, issue 1:279-87. [DOI: 10.1111/jsm.12333]

NCT01294150. The safety and effectiveness of UroLift: LIFT Pivotal Study (LIFT). clinicaltrials.gov/ct2/show/NCT01294150. https://ClinicalTrials.gov/show/NCT01294150, (first received 11 February 2011). [https://clinicaltrials.gov/ct2/show/ NCT01294150]

Rane A, McNicholas T, Woo H, Roehrborn C. 4 year results of the randomized, controlled, blinded, multi-center study for the prostatic urethral lift: the L.I.F.T. study. Journal of Endourology 2017; Vol. 30, issue Suppl 2:A105-6.

Rashid P. Multi-center prospective study of the prostatic urethral lift with two year durability. BJU International 2014;113(Suppl 4):14-5. [DOI: 10.1111/bju.12618]

Rashid P, Chin P, Bolton D, Roehrborn C, McVary K. Prospective, randomised study of prostatic urethral lift: five year results. BJU International 2017;119(Suppl 2):45. [DOI: 10.1111/jsm.12333]

Rashid P, Chin P, Bolton D, Rukstalis D, Giddens J, Gange S, et al. Prospective, randomised study of prostatic urethral lift (PUL) with three year results. BJU international 2015; Vol. 115, issue Suppl 4:10-1. [DOI: 10.1111/bju.13072]

Roehrborn C, Gange S, Shore N, Giddens J, Bolton D, Cowan B, et al. 5 year prospective, randomized, controlled study results on the minimally invasive prostatic urethral lift (PUL). Journal of Urology 2017; Vol. 197, issue Suppl 4:e511. [10.1016/ j.juro.2017.02.1222]

Roehrborn C, Gange S, Shore N, Giddens J, Bolton D, Cowan $B$, et al. Four year results from the largest, prospective, randomized study of prostatic urethral lift (PUL). European Urology Supplements 2016;15(3):e1077-a. [DOI: 10.1016/ S1569-9056(16)61078-3]

Roehrborn C, Gange S, Shore N, Giddens J, Bolton D, Cowan B, et al. Long term (5 year) results from the largest, prospective, randomized, controlled study of the minimally invasive prostatic urethral lift (PUL). European Urology Supplements 2017;16(3):e334-5. [DOI: 10.1016/S1569-9056(17)30258-0]

Roehrborn C, Gange S, Shore N, Giddens J, Bolton D, Cowan B, et al. Prospective, randomised, blinded study of prostatic urethral lift (PUL): four year results. BJU International 2017; Vol. 117, issue Suppl 3:19-20. [DOI: 10.1111/bju.13452]

Roehrborn C, Gange S, Shore N, Giddens J, Bolton D, Cowan B, et al. Prospective, randomized, blinded study of prostatic urethral lift (PUL): four year results. Journal of Urology 2016;195(Suppl 4):e456-7. [DOI: 10.1016/j.juro.2016.02.1469] 
Roehrborn C, Gange S, Shore N, Giddens J, Bolton D, Cowan B, et al. Three year durability of the prostatic urethral lift for BPH: Results of a prospective, multi-center, randomized study. Journal of Urology 2015; Vol. 193, issue Suppl 4:e92. [DOI: 10.1016/j.juro.2015.02.300]

Roehrborn CG, Barkin J, Gange SN, Shore ND, Giddens JL, Bolton DM, et al. Five year results of the prospective randomized controlled prostatic urethral L.I.F.T. study. Canadian Journal of Urology 2017;24(3):8802-13. [PUBMED: 28646935 ]

Roehrborn CG, Gange SN, Shore ND, Giddens JL, Bolton DM, Cowan BE, et al. Durability of the prostatic urethral lift: 2-year results of the L.I.F.T. study. Urology Practice 2015; Vol. 2, issue 1:26-32. [DOI: 10.1016/j.urpr.2014.08.001]

* Roehrborn CG, Gange SN, Shore ND, Giddens JL, Bolton DM, Cowan BE, et al. The prostatic urethral lift for the treatment of lower urinary tract symptoms associated with prostate enlargement due to benign prostatic hyperplasia: the L.I.F.T. study. Journal of Urology 2013; Vol. 190, issue 6:2161-7. [DOI: 10.1016/j.juro.2013.05.116]

Roehrborn CG, Rukstalis DB, Barkin J, Gange SN, Shore ND, Giddens JL, et al. Three year results of the prostatic urethral L.I.F.T. study. Canadian Journal of Urology 2015; Vol. 22, issue 3:7772-82. [PUBMED: 26068624]

Roehrborn CG, Rukstalis DB, Giddens JL, Gange SN, Shore ND, Bolton DM, et al. Two year durability of the prostatic urethral lift: multi-center prospective study. Journal of Urology 2014; Vol. 191, issue Suppl 4:e792-3. [DOI: 10.1016/j.juro.2014.02.2167]

Woo H. Prostatic urethral lift treats LUTS while preserving sexual function: results of a randomized controlled trial. BJU International 2014;113(Suppl 4):16-7. [DOI: 10.1111/bju.12618]

\section{References to studies excluded from this review}

Gratzke 2018 \{published data only\}

Gratzke C, Barkin J, Roehrborn C. Predictors of response to the prostatic urethral lift (PUL) treatment. European Urology, Supplements 2018;17(2):e1041. [DOI: 10.1016/ S1569-9056(18)31556-2]

\section{References to studies awaiting assessment}

\section{Pereira 2018 \{published data only\}}

NCT03043222. Innovative minimally invasive options in treatment of urinary problems related to prostate enlargement (BPH) in men. clinicaltrials.gov/show/NCT03043222 (first received 3 February 2017).

\footnotetext{
* Pereira K, Ford-Glanton S, Johar R, Xu P, Pham K, Gadani S, et al. Prostatic artery embolization (PAE) and prostatic urethral lift (PUL) procedures for symptomatic benign prostatic enlargement (BPH): a retrospective, single-center comparison of outcomes. Journal of Vascular and Interventional Radiology 2018; Vol. 29, issue 4 Supplement 1:S6. [DOI: 10.1016/ j.jvir.2018.01.010]
}

\section{Additional references}

\section{Agarwal 2014}

Agarwal A, Eryuzlu LN, Cartwright R, Thorlund K, Tammela TL, Guyatt GH, et al. What is the most bothersome lower urinary tract symptom? Individual- and population-level perspectives for both men and women. European Urology 2014;65(6):1211-7.

\section{Barry 1992}

Barry MJ, Fowler FJ Jr, O'Leary MP, Bruskewitz RC, Holtgrewe HL, Mebust WK, et al. The American Urological Association symptom index for benign prostatic hyperplasia. The Measurement Committee of the American Urological Association. Journal of Urology 1992;148(5):1549-57; discussion 1564.

\section{Barry 1995}

Barry MJ, Williford WO, Chang Y, Machi M, Jones K, WalkerCorkery $\mathrm{E}$, et al. Benign prostatic hyperplasia specific health status measures in clinical research: how much change in the American Urological Association symptom index and the benign prostatic hyperplasia impact index is perceptible to patients?. Journal of Urology 1995;154(5):1770-4.

\section{Barry 1997}

Barry MJ, Fowler FJ Jr, Bin L, Pitts JC 3rd, Harris CJ, Mulley AG Jr. The natural history of patients with benign prostatic hyperplasia as diagnosed by North American urologists. Journal of Urology 1997;157(1):10-4; discussion 14-5.

\section{Bhojani 2014}

Bhojani N, Gandaglia G, Sood A, Rai A, Pucheril D, Chang SL, et al. Morbidity and mortality after benign prostatic hyperplasia surgery: data from the American College of Surgeons national surgical quality improvement program. Journal of Endourology 2014;28(7):831-40.

\section{Brasure 2016}

Brasure M, MacDonald R, Dahm P, Olson CM, Nelson VA, Fink HA, et al. AHRQ comparative effectiveness reviews. Newer Medications for Lower Urinary Tract Symptoms Attributed to Benign Prostatic Hyperplasia: a Review. Rockville (MD): Agency for Healthcare Research and Quality (US), 2016.

\section{Chapple 2017}

Chapple C, Castro-Diaz D, Chuang YC, Lee KS, Liao L, Liu SP, et al. Prevalence of lower urinary tract symptoms in China, Taiwan, and South Korea: results from a cross-sectional, populationbased study. Advances in Therapy 2017; Vol. 34, issue 8:1953-65.

\section{Chin 2012}

Chin PT, Bolton DM, Jack G, Rashid P, Thavaseelan J, Yu RJ, et al. Prostatic urethral lift: two-year results after treatment for lower urinary tract symptoms secondary to benign prostatic hyperplasia. Urology 2012;79(1):5-11.

\section{Cornu 2010}

Cornu JN, Cussenot O, Haab F, Lukacs B. A widespread population study of actual medical management of lower urinary tract symptoms related to benign prostatic hyperplasia 
across Europe and beyond official clinical guidelines. European Urology 2010;58(3):450-6.

\section{Covidence 2013 [Computer program]}

Veritas Health Innovation. Covidence. Version accessed 16 August 2017. Melbourne, Australia: Veritas Health Innovation, 2013.

\section{Crawford 2006}

Crawford ED, Wilson SS, McConnell JD, Slawin KM, Lieber MC, Smith JA, et al. Baseline factors as predictors of clinical progression of benign prostatic hyperplasia in men treated with placebo. Journal of Urology 2006;175(4):1422-6; discussion 1426-7.

\section{Deeks 2011}

Deeks JJ, Higgins JP, Altman DG. Chapter 9: Analysing data and undertaking meta-analyses. In: Higgins JP, Green S, editor(s). Cochrane Handbook for Systematic Reviews of Interventions Version 5.1.0 (updated March 2011). The Cochrane Collaboration, 2011. Available from handbook.cochrane.org.

\section{Dindo 2004}

Dindo D, Demartines N, Clavien PA. Classification of surgical complications: a new proposal with evaluation in a cohort of 6336 patients and results of a survey. Annals of Surgery 2004;240(2):205-13.

\section{Dunphy 2015}

Dunphy C, Laor L, Te A, Kaplan S, Chughtai B. Relationship between depression and lower urinary tract symptoms secondary to benign prostatic hyperplasia. Reviews in Urology 2015;17(2):51-7.

\section{EAU 2017}

European Association of Urology. Treatment of non-neurogenic male LUTS. uroweb.org/guideline/treatment-of-nonneurogenic-male-luts/ (accessed 17 August 2017).

\section{Egan 2016}

Egan KB. The epidemiology of benign prostatic hyperplasia associated with lower urinary tract symptoms: prevalence and incident rates. Urologic Clinics of North America 2016;43(3):289-97.

\section{EndNote 2016 [Computer program]}

Clarivate Analytics. EndNote. Version 7.5. Philadelphia (PA): Clarivate Analytics, 2016.

\section{GRADEpro GDT 2015 [Computer program]}

McMaster University (developed by Evidence Prime). GRADEpro GDT. Version accessed 16 August 2017. Hamilton (ON): McMaster University (developed by Evidence Prime), 2015.

\section{Guyatt 2008}

Guyatt GH, Oxman AD, Vist GE, Kunz R, Falck-Ytter Y, Schünemann HJ, et al. GRADE: what is "quality of evidence" and why is it important to clinicians?. BMJ (Clinical Research Ed) 2008;336(7651):995-8.

\section{Guyatt 2011a}

Guyatt GH, Oxman AD, Kunz R, Brozek J, Alonso-Coello P, Rind $D$, et al. GRADE guidelines 6 . Rating the quality of evidence - imprecision. Journal of Clinical Epidemiology 2011;64(12):1283-93.

\section{Guyatt 2011b}

Guyatt G, Oxman AD, Akl EA, Kunz R, Vist G, Brozek J, et al. GRADE guidelines: 1 . Introduction - GRADE evidence profiles and summary of findings tables. Journal of Clinical Epidemiology 2011;64(4):383-94.

\section{Higgins 2002}

Higgins JP, Thompson SG. Quantifying heterogeneity in a metaanalysis. Statistics in Medicine 2002;21(11):1539-58.

\section{Higgins 2003}

Higgins JP, Thompson SG, Deeks JJ, Altman DG. Measuring inconsistency in meta-analyses. BMJ (Clinical Research Ed) 2003;327(7414):557-60.

\section{Higgins 2011a}

Higgins JP, Green S, editor(s). Cochrane Handbook for Systematic Reviews of Interventions Version 5.1.0 (updated March 2011). The Cochrane Collaboration, 2011. Available from handbook.cochrane.org.

\section{Higgins 2011b}

Higgins JP, Altman DG, Sterne JA. Chapter 8: Assessing risk of bias in included studies. In: Higgins JP, Green S, editor(s). Cochrane Handbook for Systematic Reviews of Interventions Version 5.1.0 (updated March 2011). The Cochrane Collaboration, 2011. Available from handbook.cochrane.org.

\section{Higgins 2011c}

Higgins JP, Deeks JJ, Altman DG. Chapter 16: Special topics in statistics. In: Higgins JP, Green S, editor(s). Cochrane Handbook for Systematic Reviews of Interventions Version 5.1.0 (updated March 2011). The Cochrane Collaboration, 2011. Available from handbook.cochrane.org.

\section{Homma 1997}

Homma Y, Kawabe K, Tsukamoto T, Yamanaka H, Okada K, Okajima E, et al. Epidemiologic survey of lower urinary tract symptoms in Asia and Australia using the international prostate symptom score. International Journal of Urology 1997;4(1):40-6.

\section{Jaeschke 1989}

Jaeschke R, Singer J, Guyatt GH. Measurement of health status. Ascertaining the minimal clinically important difference. Controlled Clinical Trials 1989;10(4):407-15.

\section{Johnston 2013}

Johnston BC, Patrick DL, Busse JW, Schünemann HJ, Agarwal A, Guyatt GH. Patient-reported outcomes in meta-analyses - Part 1: assessing risk of bias and combining outcomes. Health and Quality of Life Outcomes 2013;11:109.

\section{Jones 2016}

Jones P, Rajkumar GN, Rai BP, Aboumarzouk OM, Cleaveland P, Srirangam SJ, et al. Medium-term outcomes of UroLift 
(minimum 12 months follow-up): evidence from a systematic review. Urology 2016;97:20-4.

\section{Jung 2017}

Jung JH, McCutcheon KA, Reddy B, Borofsky M, Narayan V, $\mathrm{Kim} \mathrm{MH}$, et al. Prostatic urethral lift for the treatment of lower urinary tract symptoms in men with benign prostatic hyperplasia. Cochrane Database of Systematic Reviews 2017, Issue 10. [DOI: 10.1002/14651858.CD012832]

\section{Kozminski 2015}

Kozminski MA, Wei JT, Nelson J, Kent DM. Baseline characteristics predict risk of progression and response to combined medical therapy for benign prostatic hyperplasia (BPH). BJU International 2015;115(2):308-16.

\section{Lee 2017}

Lee SWH, Chan EMC, Lai YK. The global burden of lower urinary tract symptoms suggestive of benign prostatic hyperplasia: a systematic review and meta-analysis. Scientific Reports 2017;7(1):7984

\section{Leissner 1979}

Leissner KH, Tisell LE. The weight of the human prostate. Scandinavian Journal of Urology 1979;13(2):137-42.

\section{Liberati 2009}

Liberati A, Altman DG, Tetzlaff J, Mulrow C, Gøtzsche PC, loannidis JP, et al. The PRISMA statement for reporting systematic reviews and meta-analyses of studies that evaluate health care interventions: explanation and elaboration. PLOS Medicine 2009;6(7):e1000100.

\section{Magistro 2018}

Magistro G, Stief CG, Woo HH. Mini-review: what is new in UroLift?. European Urology Focus 2018;4(1):36-9.

\section{Martin 2014}

Martin S, Lange K, Haren MT, Taylor AW, Wittert G. Risk factors for progression or improvement of lower urinary tract symptoms in a prospective cohort of men. Journal of Urology 2014;191(1):130-7.

\section{McNicholas 2016}

McNicholas TA. Benign prostatic hyperplasia and new treatment options - a critical appraisal of the UroLift system. Medical Devices 2016;9:115-23.

\section{McVary 2011}

McVary KT, Roehrborn CG, Avins AL, Barry MJ, Bruskewitz RC, Donnell RF, et al. Update on AUA guideline on the management of benign prostatic hyperplasia. Journal of Urology 2011;185(5):1793-803.

\section{NICE 2015}

National Institute for Health and Care Excellence. UroLift for treating lower urinary tract symptoms of benign prostatic hyperplasia. www.nice.org.uk/guidance/mtg26 (accessed 28 September 2017).

\section{Nickel 2015}

Nickel JC, Brock GB, Herschorn S, Dickson R, Henneges C, Viktrup L. Proportion of tadalafil-treated patients with clinically meaningful improvement in lower urinary tract symptoms associated with benign prostatic hyperplasia integrated data from 1,499 study participants. BJU International 2015;115(5):815-21.

\section{Pariser 2015}

Pariser JJ, Pearce SM, Patel SG, Bales GT. National trends of simple prostatectomy for benign prostatic hyperplasia with an analysis of risk factors for adverse perioperative outcomes. Urology 2015;86(4):721-5.

\section{Perera 2015}

Perera M, Roberts MJ, Doi SA, Bolton D. Prostatic urethral lift improves urinary symptoms and flow while preserving sexual function for men with benign prostatic hyperplasia: a systematic review and meta-analysis. European Urology 2015;67(4):704-13.

\section{Rees 2015}

Rees J. Patients not $P$ values. BJU international 2015;115(5):678-9.

\section{Reich 2008}

Reich O, Gratzke C, Bachmann A, Seitz M, Schlenker B, Hermanek $P$, et al. Morbidity, mortality and early outcome of transurethral resection of the prostate: a prospective multicenter evaluation of 10,654 patients. Journal of Urology 2008;180(1):246-9.

\section{Review Manager 2014 [Computer program]}

Nordic Cochrane Centre, The Cochrane Collaboration. Review Manager 5 (RevMan 5). Version 5.3. Copenhagen: Nordic Cochrane Centre, The Cochrane Collaboration, 2014.

\section{Roehrborn 2003}

Roehrborn C, McConnell J, Barry M, Benaim E, Bruskewitz R, Blute ML, et al. American Urological Association guideline: management of benign prostatic hyperplasia (BPH). www.auanet.org/documents/education/clinical-guidance/ Benign-Prostatic-Hyperplasia.pdf (accessed 28 September 2017).

\section{Roehrborn 2008a}

Roehrborn CG. Pathology of benign prostatic hyperplasia. International Journal of Impotence Research 2008;20 Suppl 3:S11-8.

\section{Roehrborn 2008b}

Roehrborn CG. Current medical therapies for men with lower urinary tract symptoms and benign prostatic hyperplasia: achievements and limitations. Review in Urology 2008;10(1):14-25.

\section{Rosen 1997}

Rosen RC, Riley A, Wagner G, Osterloh IH, Kirkpatrick J, Mishra A. The International Index of Erectile Function (IIEF): a multidimensional scale for assessment of erectile dysfunction. Urology 1997;49(6):822-30. 


\section{Rosen 2007}

Rosen RC, Catania JA, Althof SE, Pollack LM, O'Leary M, Seftel AD, et al. Development and validation of four-item version of Male Sexual Health Questionnaire to assess ejaculatory dysfunction. Urology 2007;69(5):805-9.

\section{Sanchez-Gomez 2015}

Sanchez-Gomez LM, Polo-deSantos M, Gomez-Sancha F, Luengo-Matos S. Efficacy and safety of the UroLift ${ }^{\circledR}$ system for the treatment of benign prostate hyperplasia symptoms: systematic review. Actas Urológicas Españolas 2015;39(5):311-9.

\section{Schünemann 2011a}

Schünemann HJ, Oxman AD, Higgins JP, Vist GE, Glasziou P, Guyatt GH. Chapter 11: Presenting results and 'Summary of findings' tables. In: Higgins JP, Green S, editor(s). Cochrane Handbook for Systematic Reviews of Interventions Version 5.1.0 (updated March 2011). The Cochrane Collaboration, 2011. Available from handbook.cochrane.org.

\section{Schünemann 2011b}

Schünemann HJ, Oxman AD, Vist GE, Higgins JP, Deeks JJ, Glasziou P, et al. Chapter 12: Interpreting results and drawing conclusions. In: Higgins JP, Green S, editor(s). Cochrane Handbook for Systematic Reviews of Interventions Version 5.1.0 (updated March 2011). The Cochrane Collaboration, 2011. Available from handbook.cochrane.org.

\section{Schünemann 2013}

Schünemann HJ, Tugwell P, Reeves BC, Akl EA, Santesso N, Spencer FA, et al. Non-randomized studies as a source of complementary, sequential or replacement evidence for randomized controlled trials in systematic reviews on the effects of interventions. Research Synthesis Methods 2013;4(1):49-62.

\section{Spaliviero 2010}

Spaliviero M, Strom KH, Gu X, Araki M, Culkin DJ, Wong C. Does Greenlight HPS $\left.{ }^{(\mathrm{T} M}\right)$ laser photoselective vaporization

\section{CHARACTERISTICS OF STUDIES}

Characteristics of included studies [ordered by study ID] prostatectomy affect sexual function?. Journal of Endourology 2010;24(12):2051-7.

\section{Sterne 2016}

Sterne JA, Hernan MA, Reeves BC, Savovic J, Berkman ND, Viswanathan M, et al. ROBINS-I: a tool for assessing risk of bias in non-randomised studies of interventions. $B M J$ 2016;355:i4919.

\section{Strope 2015}

Strope SA, Vetter J, Elliott S, Andriole GL, Olsen MA. Use of medical therapy and success of laser surgery and transurethral resection of the prostate for benign prostatic hyperplasia. Urology 2015;86(6):1115-22.

\section{Walsh 2017}

Walsh LP. State of the art: advanced techniques for prostatic urethral lift for the relief of prostate obstruction under local anesthesia. Canadian Journal of Urology 2017;24(3):8859-64.

\section{WHO 2002}

World Health Organization. Proposed working definition of an older person in Africa for the MDS project. www.who.int/ healthinfo/survey/ageingdefnolder/en (accessed 17 August 2017).

Woo 2012

Woo HH, Bolton DM, Laborde E, Jack G, Chin PT, Rashid P, et al. Preservation of sexual function with the prostatic urethral lift: a novel treatment for lower urinary tract symptoms secondary to benign prostatic hyperplasia. Journal of Sexual Medicine 2012;9(2):568-75.

\section{Yoo 2012}

Yoo TK, Cho HJ. Benign prostatic hyperplasia: from bench to clinic. Korean Journal of Urology 2012;53(3):139-48.

* Indicates the major publication for the study

\section{Gratzke 2017}

Methods Study design: prospective, randomized, controlled, non-blinded study

Setting/country: 10 centres in Europe (Denmark, the UK, Germany)

Dates when study was conducted: February 2012 to October 2013

Participants

Inclusion criteria: men aged $\geq 50$ years with IPSS $>12, \mathrm{Q}_{\max } \leq 15 \mathrm{~mL} /$ second for $125 \mathrm{~mL}$ voided volume, postvoid residual volume $<350 \mathrm{~mL}$, prostate volume $\leq 60 \mathrm{~mL}$ on ultrasound, sexually active within 6 months before the index procedure, Sexual Health Inventory for Men score $>6$, positive response to MSHQ-EjD (excluding the response "Could not ejaculate"), Incontinence Severity Index score $\leq 4$

Exclusion criteria: active urinary tract infection at time of treatment, bacterial prostatitis within 1 year of the index procedure, cystolithiasis within 3 months of the index procedure, obstructive median lobe as assessed via ultrasound and cystoscopy, current urinary retention, urethral conditions that may prevent insertion of a rigid $20 \mathrm{~F}$ cystoscope, previous TURP or laser procedure, pelvic surgery or irradia- 
tion, PSA $\geq 10 \mathrm{ng} / \mathrm{L}$, history of prostate or bladder cancer, severe cardiac comorbidities, anticoagulants within 3 days of the index procedure (excluding up to $100 \mathrm{mg}$ aspirin (acetylsalicylic acid), other medical condition or comorbidity contraindicative for TURP or PUL, unwilling to report sexual function

Total number of participants randomly assigned: 91

Group A (PUL)

- Number of all participants randomly assigned: 45

- Age (mean $\pm S D): 63 \pm 6.8$ years

- Prostate volume (mean $\pm \mathrm{SD}$ ): $38 \pm 12 \mathrm{~mL}$

- $P S A($ mean $\pm \mathrm{SD}): 2.4 \pm 1.8 \mathrm{ng} / \mathrm{mL}$

- IPSS (mean \pm SD): $22 \pm 5.7$

- $Q_{\max }(\operatorname{mean} \pm \mathrm{SD}): 9.2 \pm 3.5 \mathrm{~mL} /$ second

\section{Group B (TURP)}

- Number of all participants randomly assigned: 46

- Age (mean \pm SD): $65 \pm 6.4$ years

- Prostate volume (mean \pm SD): $41 \pm 13 \mathrm{~mL}$

- $P S A($ mean $\pm S D): 2.6 \pm 2.1 \mathrm{ng} / \mathrm{mL}$

- IPSS (mean \pm SD): $23 \pm 5.9$

- $\mathrm{Q}_{\max }(\operatorname{mean} \pm \mathrm{SD}): 9.5 \pm 3.2 \mathrm{~mL} / \mathrm{s}$

PUL involved transurethral placement of small, permanent UroLift implants to retract the lateral lobes of the prostate and reduce obstruction. Typically, multiple implants are placed to deobstruct the prostatic urethra. Surgeons' experiences with PUL varied from 0 to 20 procedures before enrolment.

\section{Group B: TURP}

Licensed urologists trained and experienced in TURP conducted procedures in accordance with their own normal standards and practices.

Follow-up: 24 months

- IPSS

- Sexual Health Inventory for Men

- MSHQ-EjD

- Incontinence Severity Index

- Quality of Recovery visual analogue score

How measured: questionnaires

Time points measured: at baseline, 2 weeks, 1 month, 3 months, 6 months, 1 year, and 2 years

Time points reported: at baseline, 2 weeks, 1 month, 3 months, 6 months, 1 year, and 2 years

\section{Secondary outcomes}

- PGI-I questionnaire

- SF-12

- Derivative single-index SF-6D utility score

- Jenkins Sleep Questionnaire

How measured: questionnaires

Time points measured: at baseline, 2 weeks, 1 month, 3 months, 6 months, 1 year, and 2 years 
Time points reported: PGI-I questionnaire, the SF-12 at 24 months/derivative single-index SF-6D utility score, Jenkins sleep questionnaire at baseline, 2 weeks, 1 month, 3 months, 6 months, 1 year, and 2 years

\section{Safety outcomes}

How measured: Clavien-Dindo classification of adverse events

Time points measured: not reported

Time points reported: at 1 year

Subgroup: none

\begin{tabular}{|c|c|}
\hline Funding sources & Drs Speakman, Berges, Sievert, and Sønksen reported grants from NeoTract, Inc. \\
\hline Declarations of interest & $\begin{array}{l}\text { Dr Gratzke reported honoraria from Astellas, Lilly, Janssen, and Amgen. Dr Barber reported support } \\
\text { from NeoTract, Inc., Olympus, Boston Scientific, and Intuitive Surgical for proctoring and lecturing. } \\
\text { Dr Chapple reported personal fees and non-financial support from Allergan, grants, personal fees and } \\
\text { non-financial support from Astellas, personal fees and non-financial support from Boston, personal } \\
\text { fees and non-financial support from Medtronic, personal fees from Pfizer, personal fees and non-finan- } \\
\text { cial support from Recordati, and grants from NeoTract, Inc. during the conduct of the study. Dr Sonksen } \\
\text { reported support from NeoTract, Inc. for proctoring and lecturing. }\end{array}$ \\
\hline
\end{tabular}

Notes

Protocol: NCT01533038

Language of publication: English

\section{Risk of bias}

\begin{tabular}{|c|c|c|}
\hline Bias & Authors' judgement & Support for judgement \\
\hline $\begin{array}{l}\text { Random sequence genera- } \\
\text { tion (selection bias) }\end{array}$ & Low risk & $\begin{array}{l}\text { Quote: "Parallel 1:1 randomization was performed using permuted blocks of } \\
\text { random sizes, stratified by study site." }\end{array}$ \\
\hline $\begin{array}{l}\text { Allocation concealment } \\
\text { (selection bias) }\end{array}$ & Low risk & $\begin{array}{l}\text { Quote: "concealed through a password-protected computer system," "ran- } \\
\text { dom sequence revealed at the time of the procedure." }\end{array}$ \\
\hline $\begin{array}{l}\text { Blinding of participants } \\
\text { and personnel (perfor- } \\
\text { mance bias) } \\
\text { Subjective outcomes }\end{array}$ & High risk & Judgement: non-blinded study. \\
\hline $\begin{array}{l}\text { Blinding of outcome as- } \\
\text { sessment (detection bias) } \\
\text { Subjective outcomes }\end{array}$ & High risk & Judgement: non-blinded study. \\
\hline $\begin{array}{l}\text { Blinding of outcome as- } \\
\text { sessment (detection bias) } \\
\text { Objective outcomes }\end{array}$ & Low risk & Judgement: objective outcomes were not likely affected by lack of blinding. \\
\hline $\begin{array}{l}\text { Incomplete outcome data } \\
\text { (attrition bias) } \\
\text { Urological symptom } \\
\text { scores/QoL }\end{array}$ & Unclear risk & $\begin{array}{l}\text { Short term: } 40 / 45 \text { ( } 88.8 \%) \text { of randomized participants in PUL and 32/35 } \\
(91.4 \%) \text { in TURP groups were included in analysis. } \\
\text { Long term: } 37 / 45 \text { ( } 82.2 \%) \text { of randomized participants in PUL and 32/35 (91.4\%) } \\
\text { in TURP groups were included in analysis. }\end{array}$ \\
\hline $\begin{array}{l}\text { Incomplete outcome data } \\
\text { (attrition bias) }\end{array}$ & Low risk & Judgement: all participants who were randomized were included in analyses. \\
\hline
\end{tabular}


Gratzke 2017 (Continued)

Major adverse events/mi-

nor adverse events

\begin{tabular}{|c|c|c|}
\hline $\begin{array}{l}\text { Incomplete outcome data } \\
\text { (attrition bias) } \\
\text { Retreatment }\end{array}$ & Low risk & $\begin{array}{l}\text { Judgement: } 44 / 45 \text { (97.7\%) of randomized participants in PUL and 35/35 } \\
(100 \%) \text { in TURP groups were included in analysis. }\end{array}$ \\
\hline
\end{tabular}

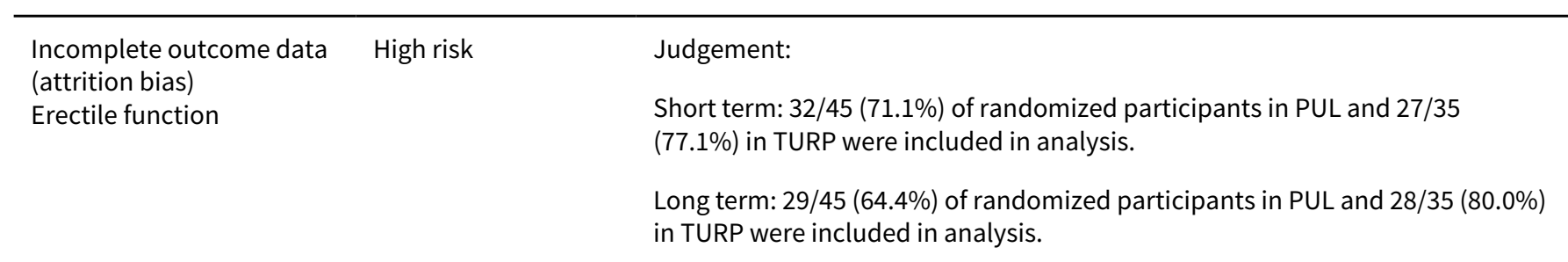

\begin{tabular}{|c|c|c|}
\hline $\begin{array}{l}\text { Incomplete outcome data } \\
\text { (attrition bias) } \\
\text { Ejaculatory function }\end{array}$ & High risk & $\begin{array}{l}\text { Judgement: } \\
\text { Short term: } 32 / 45 \text { ( } 71.1 \%) \text { of randomized participants in PUL and } 27 / 35 \\
(77.1 \%) \text { in TURP were included in analysis. }\end{array}$ \\
\hline & & $\begin{array}{l}\text { Long term: } 29 / 45(64.4 \%) \text { of randomized participants in PUL and } 27 / 35(77.1 \%) \\
\text { in TURP were included in analysis. }\end{array}$ \\
\hline
\end{tabular}

\begin{tabular}{|c|c|c|}
\hline $\begin{array}{l}\text { Incomplete outcome data } \\
\text { (attrition bias) } \\
\text { Acute urinary retention }\end{array}$ & Low risk & $\begin{array}{l}\text { Judgement: } 44 / 45(97.7 \%) \text { of randomized participants in PUL and 35/35 } \\
(100 \%) \text { in TURP were included in analysis. }\end{array}$ \\
\hline $\begin{array}{l}\text { Incomplete outcome data } \\
\text { (attrition bias) } \\
\text { Indwelling catheter }\end{array}$ & Unclear risk & Judgement: not described in the study or protocol. \\
\hline $\begin{array}{l}\text { Incomplete outcome data } \\
\text { (attrition bias) } \\
\text { Hospital stay }\end{array}$ & Low risk & $\begin{array}{l}\text { Judgement: } 44 / 45(97.7 \%) \text { of randomized participants in PUL and 35/35 } \\
(100 \%) \text { in TURP were included in analysis. }\end{array}$ \\
\hline $\begin{array}{l}\text { Selective reporting (re- } \\
\text { porting bias) }\end{array}$ & Low risk & $\begin{array}{l}\text { Judgement: review outcomes were prespecified in the protocol } \\
\text { (NCT01533038) and were analyzed as planned. }\end{array}$ \\
\hline Other bias & Low risk & Judgement: not detected. \\
\hline
\end{tabular}

Roehrborn 2013

\begin{tabular}{ll} 
Methods & Study design: multicentre randomized blinded trial \\
Setting/country: 19 centres in US 14, Canada 2, Australia 3 \\
Dates when study was conducted: February to December 2011 \\
\hline Participants & $\begin{array}{l}\text { Inclusion criteria: men aged } \geq 50 \text { years, provided informed consent, had no prior surgical treatment } \\
\text { for BPH, and were required to undergo washouts of } 2 \text { weeks for alpha-blocker, } 3 \text { months for } 5 a-r e d u c- \\
\text { tase inhibitor, and } 3 \text { days for anticoagulants. Admission to the study required } \geq \text { IPSS } 13, \mathrm{Q}_{\mathrm{max}} \leq 12 \mathrm{~mL} / \\
\text { second with a } 125 \mathrm{~mL} \text { voided volume and a } 30 \text { - to } 80 \text { - } \mathrm{mL} \text { prostate volume }\end{array}$
\end{tabular}

Exclusion criteria: median lobe obstruction, retention, postvoid residual volume $>250 \mathrm{~mL}$, active infection, PSA $>10 \mathrm{ng} / \mathrm{mL}$ (unless negative biopsy), cystolithiasis within 3 months, and bacterial prostatitis within 1 year

\section{Total number of participants randomly assigned: 206}


Roehrborn 2013 (Continued)

\section{Group A (PUL)}

- Number of all participants randomly assigned: 140

- Age (mean \pm SD): $67 \pm 8.6$ years

- Prostate volume (mean \pm SD): $44.5 \pm 12.4 \mathrm{~mL}$

- $P S A(m e a n \pm S D): 2.4 \pm 2.0 \mathrm{ng} / \mathrm{mL}$

- IPSS (mean \pm SD): $22.2 \pm 5.48$

- $\mathrm{Q}_{\max }(\operatorname{mean} \pm \mathrm{SD}): 8.9 \pm 2.2 \mathrm{~mL} /$ second

Group B (Sham)

- Number of all participants randomly assigned: 66

- Age (mean \pm SD): $65 \pm 8.0$ years

- Prostate volume (mean \pm SD): $40.9 \pm 10.8 \mathrm{~mL}$

- PSA (mean $\pm \mathrm{SD}): 2.1 \pm 1.6 \mathrm{ng} / \mathrm{mL}$

- IPSS: $24.4 \pm 5.75$

- $\mathrm{Q}_{\max }(\operatorname{mean} \pm \mathrm{SD}): 8.8 \pm 2.2 \mathrm{~mL} /$ second

Transprostatic adjustable UroLift implants are permanently implanted to retract obstructing lateral lobes and expand the urethral lumen. After rigid cystoscopy is performed, the implant delivery device is inserted into the $20-\mathrm{F}$ sheath. Under cystoscopic visualization using a $2.9 \mathrm{~mm} 0$-degree lens, the delivery device is angled anterolaterally to compress the obstructive lobe. A 19-gauge needle, housing a monofilament with metallic tab, is then deployed through the prostate lobe. As the needle is retracted, the tab engages the prostate capsule and the monofilament is tensioned. Finally, the urethral endpiece is attached to the monofilament, which is then cut, delivering the in situ-sized implant.

Group B: sham

Conducted with as similar an experience as possible to PUL.

Follow-up: 3 months

\section{Primary outcome}

- Reduction in IPSS at 3 months after the PUL procedure was $\geq 25 \%$ greater than that of sham

How measured: IPSS questionnaires

Time points measured: at baseline, 2 weeks, 1 month, and 3 months

Time points reported: at baseline, 2 weeks, 1 month, and 3 months

\section{Secondary outcome}

- QoL (IPSS-QoL)

- BPH Impact Index

- International Index of Erectile Function

- MSHQ-EjD

How measured: questionnaires

Time points measured: at baseline, 2 weeks, 1 month, and 3 months

Time points reported: at baseline, 2 weeks, 1 month, and 3 months

\section{Safety outcomes}

How measured: adverse events

Time points measured: not reported 
Roehrborn 2013 (Continued)

Time points reported: at 3 months

Subgroup: none

\begin{tabular}{ll}
\hline Funding sources & NeoTract, Fe/Male Health Centre \\
\hline Declarations of interest & NeoTract, Fe/Male Health Centre \\
\hline Notes & Protocol: NCT01294150 \\
& Language of publication: English \\
\hline
\end{tabular}

\section{Risk of bias}

\begin{tabular}{|c|c|c|}
\hline Bias & Authors' judgement & Support for judgement \\
\hline $\begin{array}{l}\text { Random sequence genera- } \\
\text { tion (selection bias) }\end{array}$ & Low risk & $\begin{array}{l}\text { Quote: "Randomization was conducted just before treatment using permut- } \\
\text { ed blocks of various sizes chosen at random through a central electronic data } \\
\text { program." }\end{array}$ \\
\hline $\begin{array}{l}\text { Allocation concealment } \\
\text { (selection bias) }\end{array}$ & Low risk & $\begin{array}{l}\text { Quote: "concealed through password protected electronic database pro- } \\
\text { gram." }\end{array}$ \\
\hline $\begin{array}{l}\text { Blinding of participants } \\
\text { and personnel (perfor- } \\
\text { mance bias) } \\
\text { Subjective outcomes }\end{array}$ & High risk & $\begin{array}{l}\text { Judgement: we contacted with author and they clarified the blinding of partic- } \\
\text { ipants and outcome assessor. The personnel were not blinded. }\end{array}$ \\
\hline $\begin{array}{l}\text { Blinding of outcome as- } \\
\text { sessment (detection bias) } \\
\text { Subjective outcomes }\end{array}$ & Low risk & $\begin{array}{l}\text { Quote: "An independent data monitoring committee assessed safety, and all } \\
\text { AEs were adjudicated and assessed by an independent clinical events commit- } \\
\text { tee... A double-blind was maintained through the 3-month end point with the } \\
\text { patient and questionnaire administrator blinded to randomization. Blinding of } \\
\text { participants was tested upon discharge and at each follow-up to } 3 \text { months." }\end{array}$ \\
\hline
\end{tabular}

Blinding of outcome as- Low risk Judgement: objective outcomes were not likely affected by lack of blinding.

sessment (detection bias)

Objective outcomes

Incomplete outcome data Low risk Judgement: all participants who were randomized were included in analysis.
(attrition bias)
Urological symptom
scores/QoL

\begin{tabular}{l}
\hline Incomplete outcome data Low risk Judgement: all participants who were randomized were included in analysis. \\
(attrition bias) \\
Major adverse events/mi- \\
nor adverse events
\end{tabular}

Incomplete outcome data Low risk Judgement: all participants who were randomized were included in analysis.
(attrition bias)
Retreatment

\begin{tabular}{lll}
\hline $\begin{array}{l}\text { Incomplete outcome data } \\
\text { (attrition bias) }\end{array}$ & Low risk \\
$\begin{array}{l}\text { Erectile function } \\
\text { Incomplete outcome data }\end{array}$ & High risk & $\begin{array}{l}\text { Judgement: 132/140 (94.2\%) of randomized participants in PUL and 65/66 } \\
(98.4 \%) \text { in sham groups were included in the analysis. }\end{array}$ \\
$\begin{array}{l}\text { (attrition bias) } \\
\text { Ejaculatory function }\end{array}$ & $\begin{array}{l}\text { Judgement: } 94 / 140(67.1 \%) \text { of randomized participants in PUL and 50/66 } \\
(75.7 \%) \text { in sham groups were included in analysis. }\end{array}$ \\
\hline \hline
\end{tabular}


Roehrborn 2013 (Continued)

Incomplete outcome data Low risk Judgement: all participants who were randomized were included in analysis. (attrition bias)

Acute urinary retention

Incomplete outcome data Unclear risk Judgement: not described in the study or protocol.
(attrition bias)

Indwelling catheter

\begin{tabular}{ll}
\hline $\begin{array}{l}\text { Incomplete outcome data } \\
\text { (attrition bias) }\end{array}$ & Low risk \\
\end{tabular}

Hospital stay

\begin{tabular}{lll}
\hline $\begin{array}{l}\text { Selective reporting (re- } \\
\text { porting bias) }\end{array}$ & Low risk & $\begin{array}{l}\text { Judgement: review outcomes were prespecified in the protocol } \\
\text { (NCT01294150) and were analyzed as planned. }\end{array}$ \\
\hline Other bias & Low risk & Judgement: not detected. \\
\hline
\end{tabular}

BPH: benign prostatic hyperplasia; IPSS: International Prostate Symptom Score; MSHQ-EjD: Male Sexual Health Questionnaire for Ejaculatory Dysfunction; PGI-I: Patient Global Impression of Improvement; PSA: prostate-specific antigen; PUL: prostatic urethral lift; $\mathrm{Q}_{\text {max: }}$ maximum flow rate; QoL: quality of life; SD: standard deviation; SF-6D: Short-Form Six-Dimension; SF-12: 12-item Short-Form Health Survey; TURP: transurethral resection of prostate.

Characteristics of excluded studies [ordered by study ID]

\begin{tabular}{ll}
\hline Study & Reason for exclusion \\
\hline Gratzke 2018 & Wrong study design (single arm) \\
\hline
\end{tabular}

\section{Characteristics of studies awaiting assessment [ordered by study ID]}

\section{Pereira 2018}

Methods Study design: retrospective non-randomized parallel assignment (open label)

Setting/country: single-centre in US

Dates when study was conducted: not reported

\section{Participants}

Inclusion criteria: men aged $\geq 40$ years; willing, able, and mentally competent to provide written informed consent; willing to comply with all study procedures; and be available for the duration of the study with a diagnosis of urinary symptoms from prostatic enlargement refractory to medical therapy for $\geq 6$ months (IPSS $>12$ ).

Exclusion criteria: men with:

- PAE

- Active urinary tract infections, prostatitis, or interstitial cystitis

- Biopsy-confirmed prostate, bladder, or urethral cancer

- The following patients must undergo prostate biopsy with a minimum of 12 cores and have a negative histopathology report to be enrolled in the study:

- digital rectal examination findings suspicious for prostate cancer

- baseline PSA levels $>10 \mathrm{ng} / \mathrm{mL}$

- baseline PSA levels $>2.5 \mathrm{ng} / \mathrm{mL}$ and $<10 \mathrm{ng} / \mathrm{mL}$ and free PSA $<25 \%$ of total PSA

- cystoscopy findings suspicious for bladder cancer must undergo biopsy 
- Unable to have computer tomography angiography of the prostate imaging

- Bladder atonia, neurogenic bladder disorder, or other neurological disorder that is impacting bladder function (e.g. multiple sclerosis, Parkinson's disease, spinal cord injuries, etc.)

- Urethral stricture, bladder neck contracture, sphincter abnormalities, bladder diverticulum, urinary obstruction due to causes other than $\mathrm{BPH}$, or other potentially confounding bladder or urethral disease or condition

- Acontractile detrusor.

- Known upper tract renal disease

- Cystolithiasis

- Any known condition that limits catheter-based intervention or is a contraindication to embolization, such as intolerance to a vessel occlusion procedure or severe atherosclerosis. Known major iliac arterial occlusive disease

- American Society of Anesthesiologists classification $>3$ or severe medical debilitating condition

- Baseline serum creatinine level $>1.8 \mathrm{mg} / \mathrm{dL}$

- Previous rectal surgery other than haemorrhoidectomy, or history of rectal disease

- History of pelvic irradiation or radical pelvic surgery

- Allergy to iodinated contrast agents

PUL

- Active urinary tract infections, prostatitis, or interstitial cystitis

- Biopsy-confirmed prostate, bladder, or urethral cancer

- The following men must undergo prostate biopsy with a minimum of 12 cores and have a negative histopathology report to be enrolled in the study:

- digital rectal examination findings suspicious for prostate cancer

- baseline PSA levels $>10 \mathrm{ng} / \mathrm{mL}$

- baseline PSA levels $>2.5 \mathrm{ng} / \mathrm{mL}$ and $<10 \mathrm{ng} / \mathrm{mL}$ and free PSA $<25 \%$ of total PSA

- cystoscopy findings suspicious for bladder cancer must undergo biopsy

- Significant median lobe enlargement

- Large prostates, volume $>80 \mathrm{~g}$

- Bladder atonia, neurogenic bladder disorder, or other neurological disorder that is impacting bladder function (e.g. multiple sclerosis, Parkinson's disease, spinal cord injuries, etc.)

- Urethral stricture, bladder neck contracture, sphincter abnormalities, bladder diverticulum, urinary obstruction due to causes other than BPH, or other potentially confounding bladder or urethral disease or condition

- Urethral conditions that may prevent insertion of a rigid 20-F cystoscope

- Current urinary retention

- Acontractile detrusor

- Current gross haematuria

- Known upper tract renal disease

- Cystolithiasis

- American Society of Anesthesiologists classification $>3$ or severe medical debilitating condition

- History of pelvic irradiation or radical pelvic surgery

- Known allergy to nickel

Total number of participants analyzed: 37

Group A (PAE)

- Number of all participants analyzed: 21

- Age: not reported

- Prostate volume: not reported

- PSA: not reported

- IPSS (mean \pm SD): $25.2 \pm 7.0$

- $\mathrm{Q}_{\max }:$ not reported 
Pereira 2018 (Continued)

$$
\begin{aligned}
& \text { Group B (PUL) } \\
& \text { - Number of all participants analyzed: } 16 \\
& \text { - Age: not reported } \\
& \text { - Prostate volume: not reported } \\
& \text { - PSA: not reported } \\
& \text { - IPSS (mean } \pm \mathrm{SD}): 21.7 \pm 8.95 \\
& \text { - } \mathrm{Q}_{\text {max }} \text { : not reported }
\end{aligned}
$$

\begin{tabular}{ll}
\hline Interventions & Group A: PAE \\
Group B: PUL & Follow-up: 6 months \\
\hline Outcomes & IPSS/QoL \\
& How measured: IPSS questionnaire \\
& Time points measured: at the baseline; 3, 6, and 12 months \\
& Time points reported: at the baseline; 3 and 6 months (preliminary data) \\
& Safety outcomes: not reported \\
& Subgroup: none \\
& Abstract only; the study is not completed. \\
& Protocol: NCT03043222 \\
Language of publication: English
\end{tabular}

BPH: benign prostatic hyperplasia; IPSS; International Prostate Symptom Score; PSA: prostate-specific antigen; PAE: prostatic arterial embolization; PUL: prostatic urethral lift; $\mathrm{Q}_{\max }$ : maximum flow rate; QoL: quality of life.

\section{DATA AND ANALYSES}

\section{Comparison 1. Prostatic urethral lift (PUL) versus sham (short term)}

\begin{tabular}{lllll}
\hline $\begin{array}{l}\text { Outcome or subgroup ti- } \\
\text { tle }\end{array}$ & No. of studies & $\begin{array}{l}\text { No. of partici- } \\
\text { pants }\end{array}$ & Statistical method & Effect size \\
\hline $\begin{array}{l}1 \text { Urological symptom } \\
\text { scores }\end{array}$ & 1 & 206 & Mean Difference (IV, Random, 95\% Cl) & $-5.20[-7.44,-2.96]$ \\
\hline 2 Quality of life & 1 & 206 & Mean Difference (IV, Random, 95\% Cl) & $-1.20[-1.67,-0.73]$ \\
\hline 3 Major adverse events & 1 & 206 & Risk Ratio (M-H, Random, 95\% Cl) & $3.3[0.41,26.28]$ \\
\hline 4 Ejaculatory function & 1 & 144 & Mean Difference (IV, Random, 95\% Cl) & $0.50[-0.38,1.38]$ \\
\hline 5 Erectile function & 1 & 197 & Mean Difference (IV, Random, 95\% Cl) & $-1.4[-3.24,0.44]$ \\
\hline 6 Minor adverse events & 1 & 206 & Risk Ratio (M-H, Random, 95\% Cl) & $1.69[1.33,2.16]$ \\
\hline
\end{tabular}




\begin{tabular}{lllll}
\hline $\begin{array}{l}\text { Outcome or subgroup ti- } \\
\text { tle }\end{array}$ & No. of studies & $\begin{array}{l}\text { No. of partici- } \\
\text { pants }\end{array}$ & Statistical method & Effect size \\
\hline 7 Acute urinary retention & 1 & 206 & Risk Ratio (M-H, Random, 95\% Cl) & $0.47[0.03,7.42]$ \\
\hline 8 Hospital stay (days) & 1 & 201 & Mean Difference (IV, Random, 95\% Cl) & $0.03[-0.09,0.15]$ \\
\hline
\end{tabular}

Analysis 1.1. Comparison 1 Prostatic urethral lift (PUL) versus sham (short term), Outcome 1 Urological symptom scores.

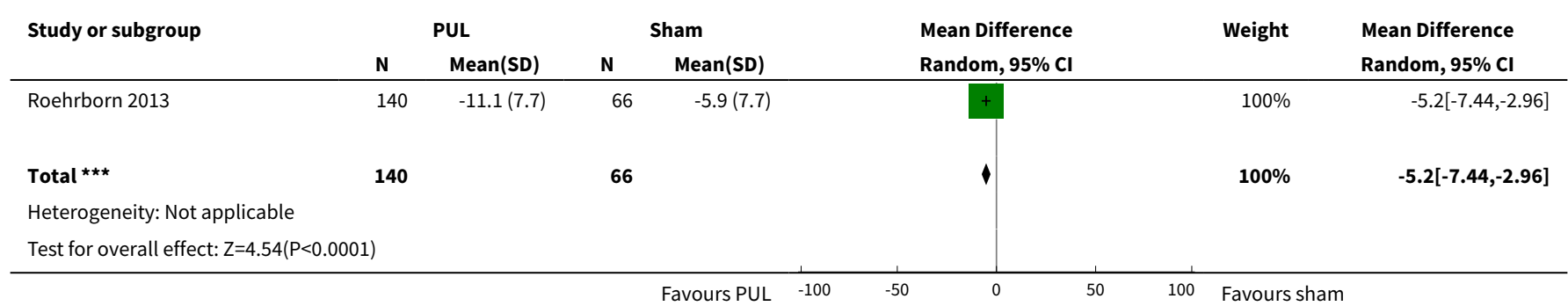

Analysis 1.2. Comparison 1 Prostatic urethral lift (PUL) versus sham (short term), Outcome 2 Quality of life.

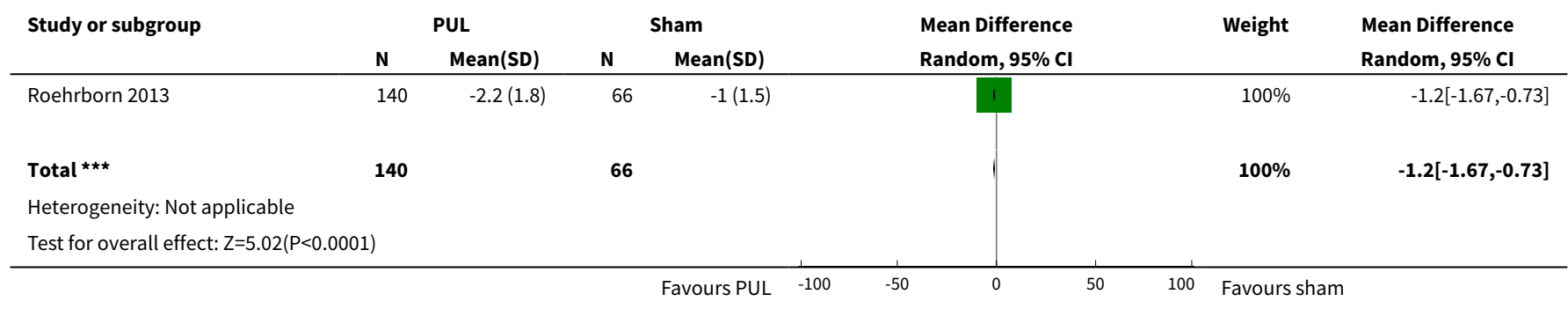

Analysis 1.3. Comparison 1 Prostatic urethral lift (PUL) versus sham (short term), Outcome 3 Major adverse events.

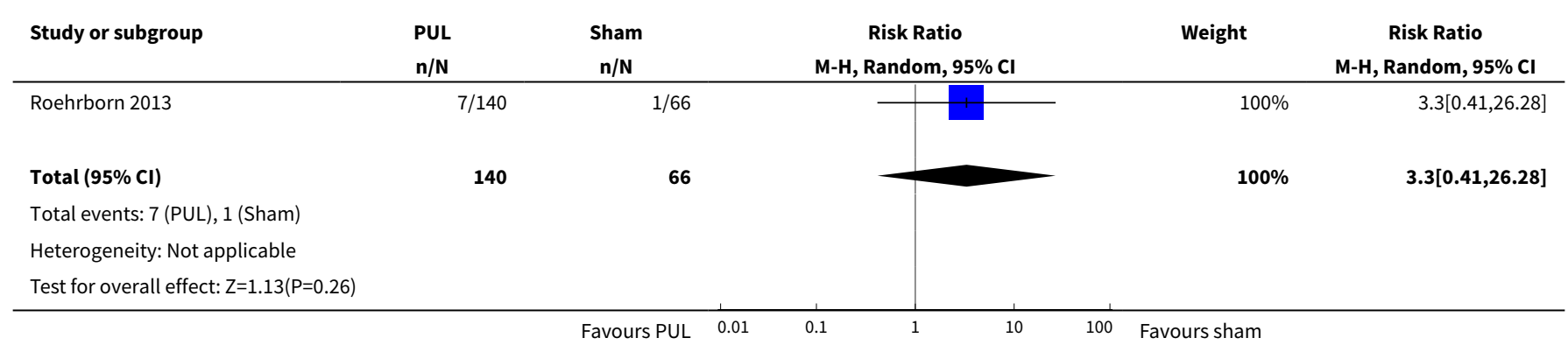


Analysis 1.4. Comparison 1 Prostatic urethral lift (PUL) versus sham (short term), Outcome 4 Ejaculatory function.

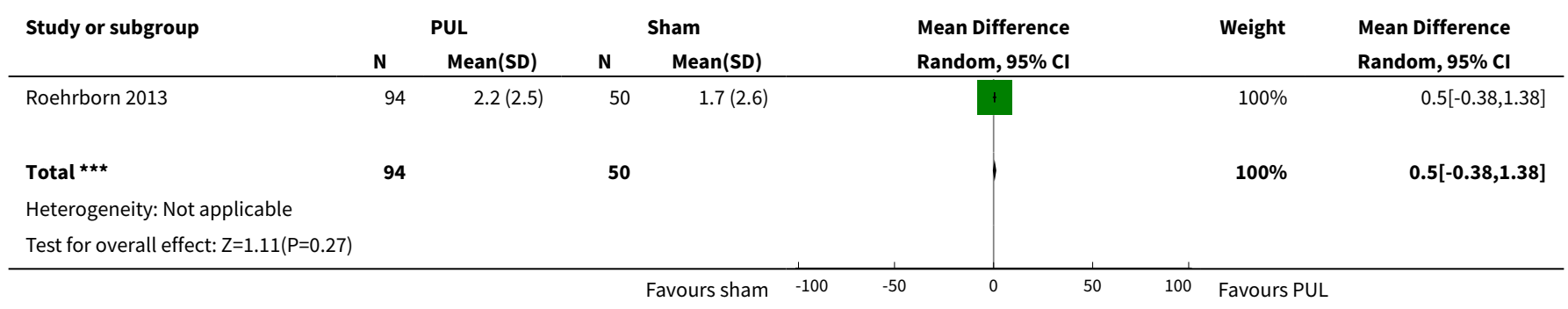

\section{Analysis 1.5. Comparison 1 Prostatic urethral lift (PUL) versus sham (short term), Outcome 5 Erectile function.}

\begin{tabular}{|c|c|c|c|c|c|c|c|}
\hline \multirow[t]{2}{*}{ Study or subgroup } & \multicolumn{2}{|c|}{ PUL } & \multicolumn{2}{|c|}{ Sham } & \multirow{2}{*}{$\begin{array}{l}\text { Mean Difference } \\
\text { Random, } 95 \% \mathrm{Cl}\end{array}$} & \multirow[t]{2}{*}{ Weight } & \multirow{2}{*}{$\begin{array}{l}\text { Mean Difference } \\
\text { Random, } 95 \% \mathrm{Cl}\end{array}$} \\
\hline & $\mathbf{N}$ & $\operatorname{Mean}(S D)$ & $\mathbf{N}$ & $\operatorname{Mean}(S D)$ & & & \\
\hline Roehrborn 2013 & 132 & $0.1(5.8)$ & 65 & $1.5(6.4)$ & + & $100 \%$ & $-1.4[-3.24,0.44]$ \\
\hline 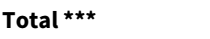 & 132 & & 65 & & 1 & $100 \%$ & $-1.4[-3.24,0.44]$ \\
\hline \multicolumn{8}{|c|}{ Heterogeneity: Not applicable } \\
\hline Test for overall effect & & & & & & & \\
\hline
\end{tabular}

Analysis 1.6. Comparison 1 Prostatic urethral lift (PUL) versus sham (short term), Outcome 6 Minor adverse events.

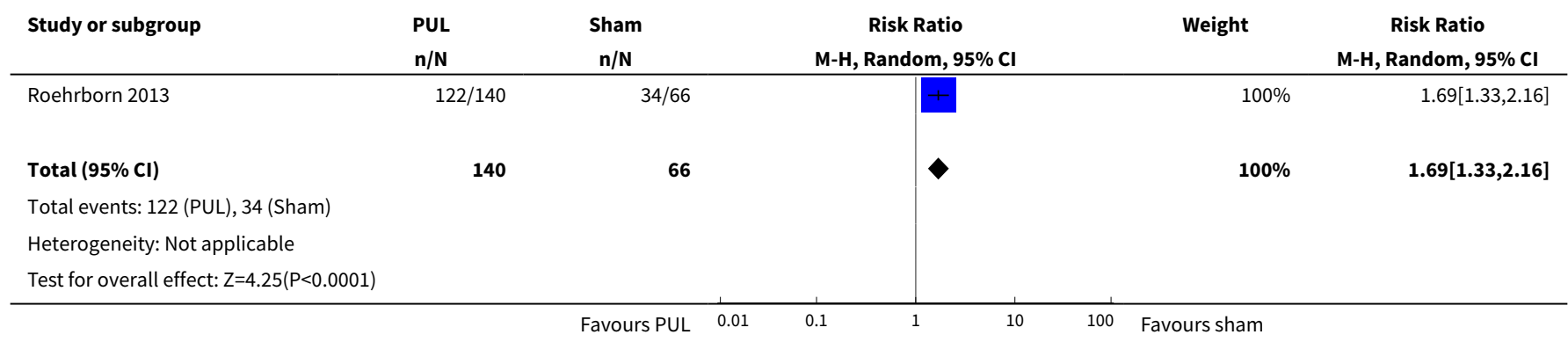

Analysis 1.7. Comparison 1 Prostatic urethral lift (PUL) versus sham (short term), Outcome 7 Acute urinary retention.




Analysis 1.8. Comparison 1 Prostatic urethral lift (PUL) versus sham (short term), Outcome 8 Hospital stay (days).

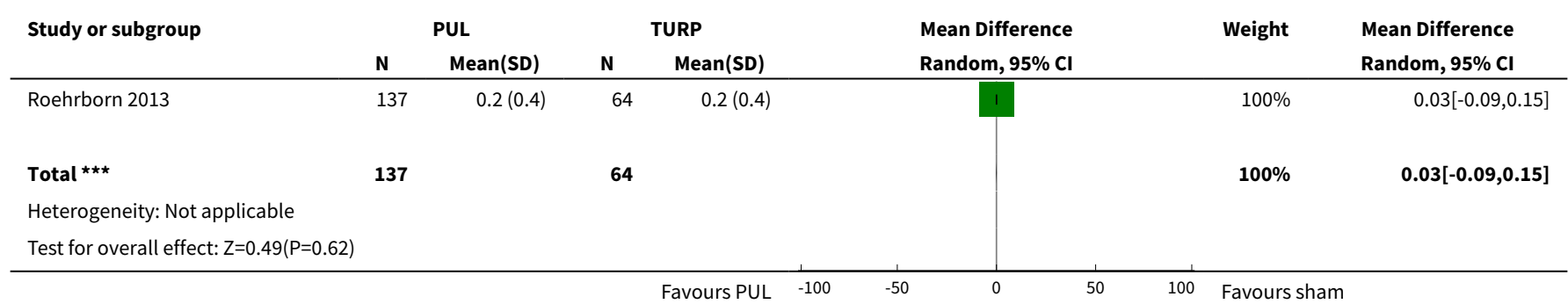

\section{Comparison 2. Prostatic urethral lift (PUL) versus transurethral resection of prostate (TURP) (short term)}

\begin{tabular}{|c|c|c|c|c|}
\hline $\begin{array}{l}\text { Outcome or subgroup ti- } \\
\text { tle }\end{array}$ & No. of studies & $\begin{array}{l}\text { No. of partici- } \\
\text { pants }\end{array}$ & Statistical method & Effect size \\
\hline $\begin{array}{l}1 \text { Urological symptom } \\
\text { scores }\end{array}$ & 1 & 72 & Mean Difference (IV, Random, 95\% CI) & $4.5[1.10,7.90]$ \\
\hline 2 Quality of life & 1 & 72 & Mean Difference (IV, Random, 95\% CI) & $0.30[-0.49,1.09]$ \\
\hline 3 Major adverse events & 1 & 79 & Risk Ratio (M-H, Random, 95\% Cl) & $0.64[0.18,2.19]$ \\
\hline 4 Retreatment & 1 & 79 & Risk Ratio (M-H, Random, 95\% Cl) & $1.19[0.21,6.75]$ \\
\hline 5 Erectile function & 1 & 59 & Mean Difference (IV, Random, 95\% CI) & $0.8[-1.50,3.10]$ \\
\hline 6 Ejaculatory function & 1 & 59 & Mean Difference (IV, Random, 95\% CI) & $5.0[3.08,6.92]$ \\
\hline 7 Minor adverse events & 1 & 79 & Risk Ratio (M-H, Random, 95\% Cl) & $0.88[0.70,1.09]$ \\
\hline 8 Acute urinary retention & 1 & 79 & Risk Ratio (M-H, Random, 95\% Cl) & $7.2[0.40,129.38]$ \\
\hline $\begin{array}{l}9 \text { Indwelling urinary } \\
\text { catheter }\end{array}$ & 1 & 79 & Risk Ratio (M-H, Random, 95\% Cl) & $0.46[0.25,0.84]$ \\
\hline 10 Hospital stay (days) & 1 & 79 & Mean Difference (IV, Random, 95\% CI) & $-0.90[-1.32,-0.48]$ \\
\hline
\end{tabular}

Analysis 2.1. Comparison 2 Prostatic urethral lift (PUL) versus transurethral resection of prostate (TURP) (short term), Outcome 1 Urological symptom scores.

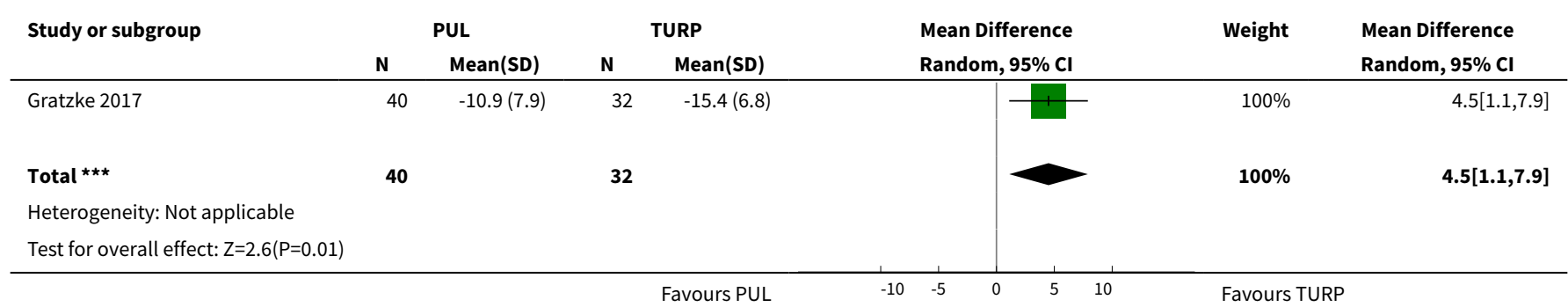


Analysis 2.2. Comparison 2 Prostatic urethral lift (PUL) versus transurethral resection of prostate (TURP) (short term), Outcome 2 Quality of life.

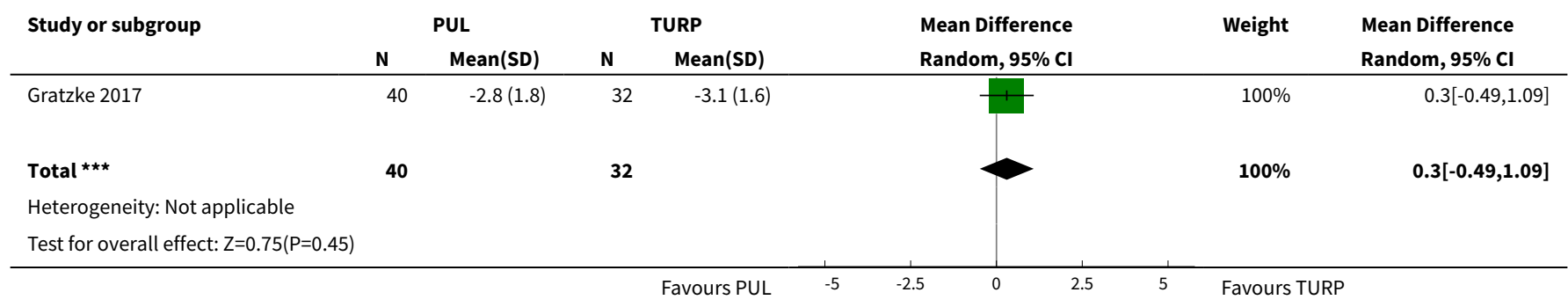

Analysis 2.3. Comparison 2 Prostatic urethral lift (PUL) versus transurethral resection of prostate (TURP) (short term), Outcome 3 Major adverse events.

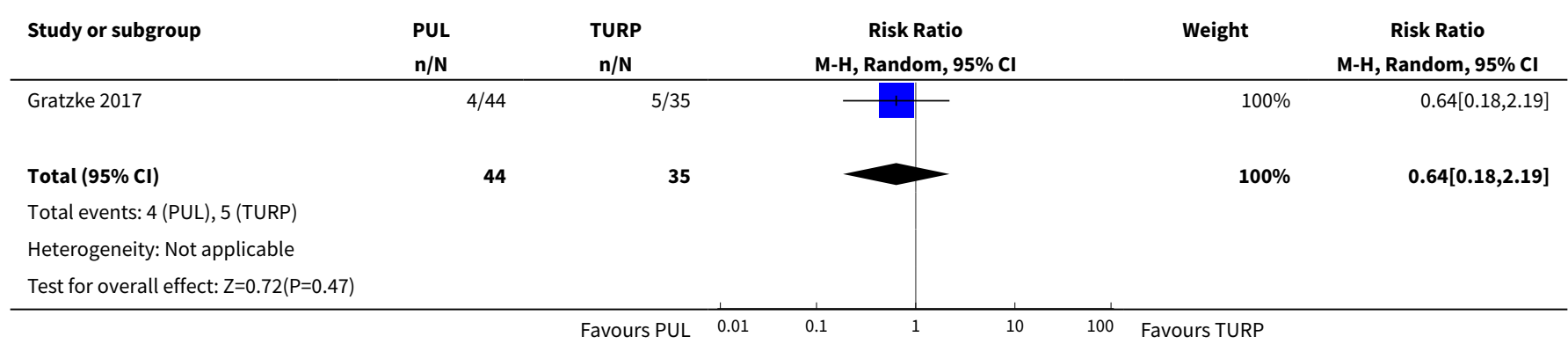

Analysis 2.4. Comparison 2 Prostatic urethral lift (PUL) versus transurethral resection of prostate (TURP) (short term), Outcome 4 Retreatment.

\begin{tabular}{|c|c|c|c|c|c|}
\hline Study or subgroup & $\begin{array}{l}\text { PUL } \\
n / N\end{array}$ & $\begin{array}{l}\text { TURP } \\
\mathbf{n} / \mathbf{N}\end{array}$ & $\begin{array}{c}\text { Risk Ratio } \\
\text { M-H, Random, } 95 \% \mathrm{CI}\end{array}$ & Weight & $\begin{array}{c}\text { Risk Ratio } \\
\text { M-H, Random, } 95 \% \mathrm{Cl}\end{array}$ \\
\hline Gratzke 2017 & $3 / 44$ & $2 / 35$ & + & $100 \%$ & $1.19[0.21,6.75]$ \\
\hline Total $(95 \% \mathrm{Cl})$ & 44 & 35 & & $100 \%$ & $1.19[0.21,6.75]$ \\
\hline \multicolumn{6}{|l|}{ Total events: 3 (PUL), 2 (TURP) } \\
\hline \multicolumn{6}{|l|}{ Heterogeneity: Not applicable } \\
\hline & & $\begin{array}{ll} & 1 \\
\text { Favours PUL } & 0.01\end{array}$ & 1 & ours TURP & \\
\hline
\end{tabular}

Analysis 2.5. Comparison 2 Prostatic urethral lift (PUL) versus transurethral resection of prostate (TURP) (short term), Outcome 5 Erectile function.

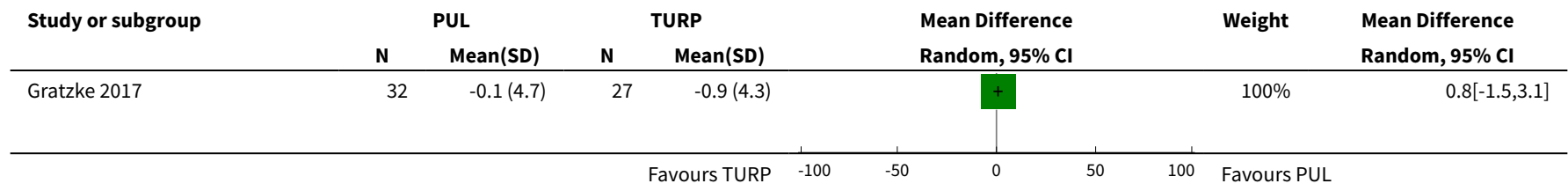




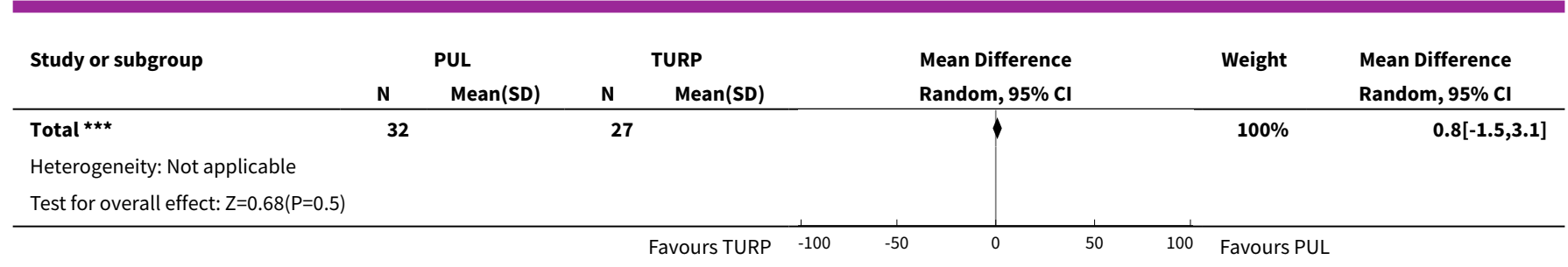

Analysis 2.6. Comparison 2 Prostatic urethral lift (PUL) versus transurethral resection of prostate (TURP) (short term), Outcome 6 Ejaculatory function.



Analysis 2.7. Comparison 2 Prostatic urethral lift (PUL) versus transurethral resection of prostate (TURP) (short term), Outcome 7 Minor adverse events.

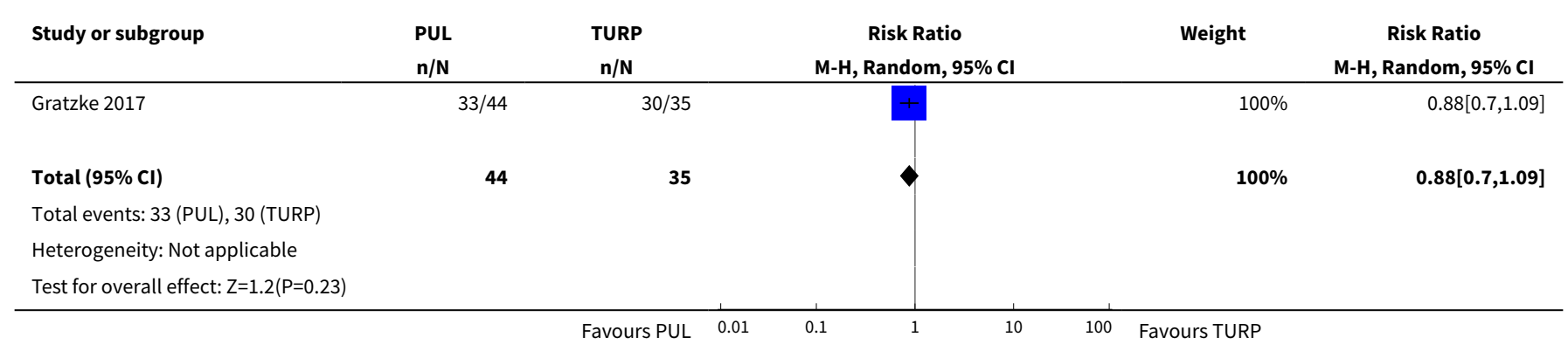

Analysis 2.8. Comparison 2 Prostatic urethral lift (PUL) versus transurethral resection of prostate (TURP) (short term), Outcome 8 Acute urinary retention.




Analysis 2.9. Comparison 2 Prostatic urethral lift (PUL) versus transurethral resection of prostate (TURP) (short term), Outcome 9 Indwelling urinary catheter.

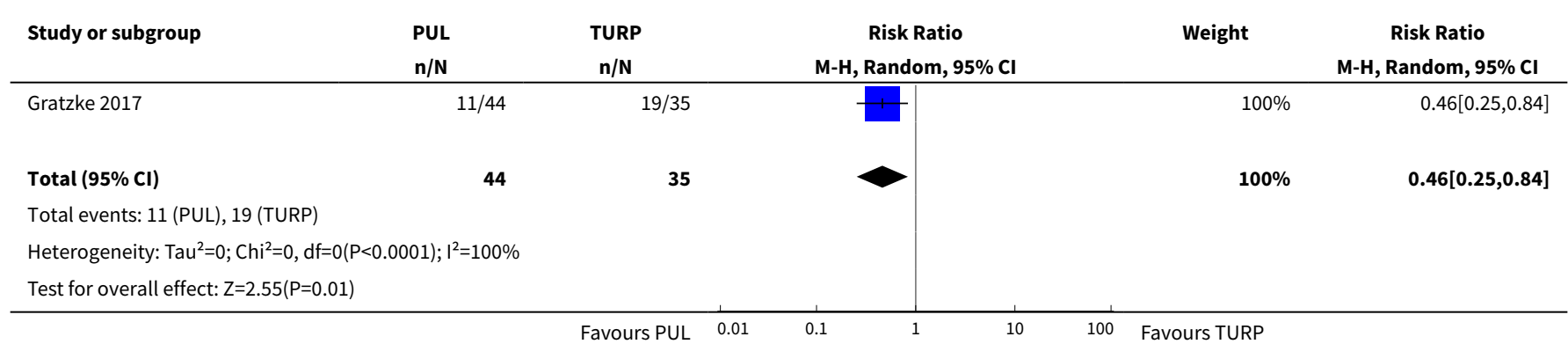

Analysis 2.10. Comparison 2 Prostatic urethral lift (PUL) versus transurethral resection of prostate (TURP) (short term), Outcome 10 Hospital stay (days).

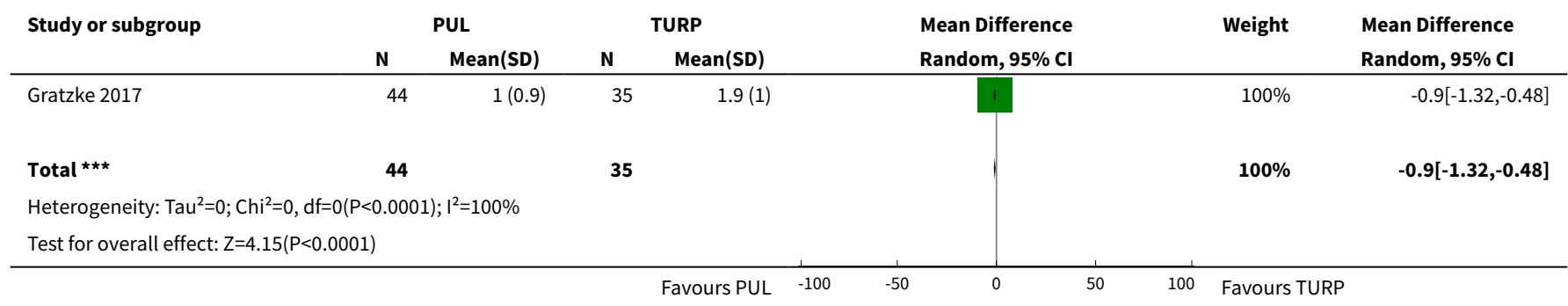

Comparison 3. Prostatic urethral lift (PUL) versus transurethral resection of prostate (TURP) (long term)

\begin{tabular}{lllll}
\hline $\begin{array}{l}\text { Outcome or subgroup } \\
\text { title }\end{array}$ & No. of studies & $\begin{array}{l}\text { No. of partici- } \\
\text { pants }\end{array}$ & Statistical method & Effect size \\
\hline $\begin{array}{l}1 \text { Urological symptom } \\
\text { scores }\end{array}$ & 1 & 69 & Mean Difference (IV, Random, 95\% CI) & $6.10[2.16,10.04]$ \\
\hline 2 Quality of life & 1 & 69 & Mean Difference (IV, Random, 95\% CI) & $0.80[-0.00,1.60]$ \\
\hline 3 Retreatment & 1 & 79 & Risk Ratio (M-H, Random, 95\% Cl) & $2.39[0.51,11.10]$ \\
\hline 4 Erectile function & 1 & 57 & Mean Difference (IV, Random, 95\% CI) & $1.60[-0.80,4.00]$ \\
\hline 5 Ejaculatory function & 1 & 56 & Mean Difference (IV, Random, 95\% Cl) & $4.3[2.17,6.43]$ \\
\hline 6 Minor adverse events & 1 & 80 & Risk Ratio (M-H, Random, 95\% Cl) & $0.92[0.72,1.17]$ \\
\hline
\end{tabular}


Analysis 3.1. Comparison 3 Prostatic urethral lift (PUL) versus transurethral resection of prostate (TURP) (long term), Outcome 1 Urological symptom scores.

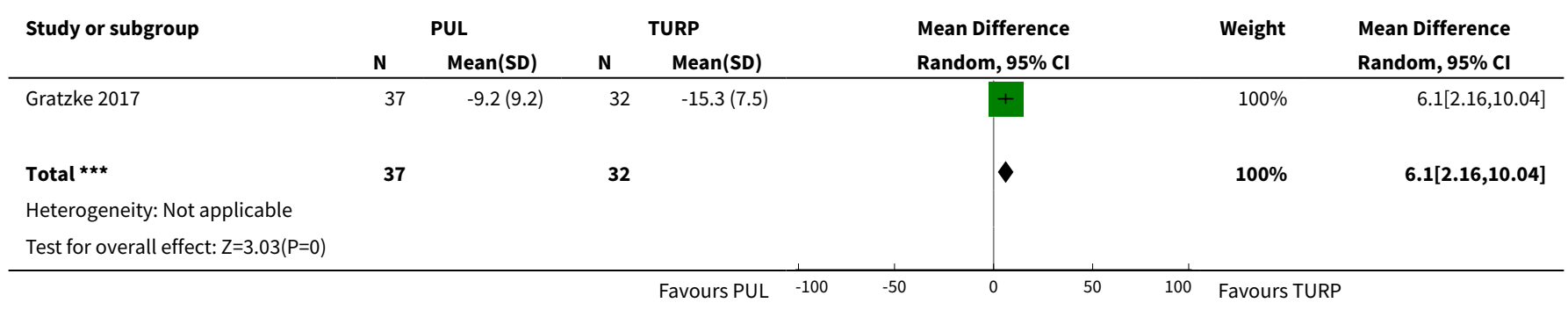

Analysis 3.2. Comparison 3 Prostatic urethral lift (PUL) versus transurethral resection of prostate (TURP) (long term), Outcome 2 Quality of life.

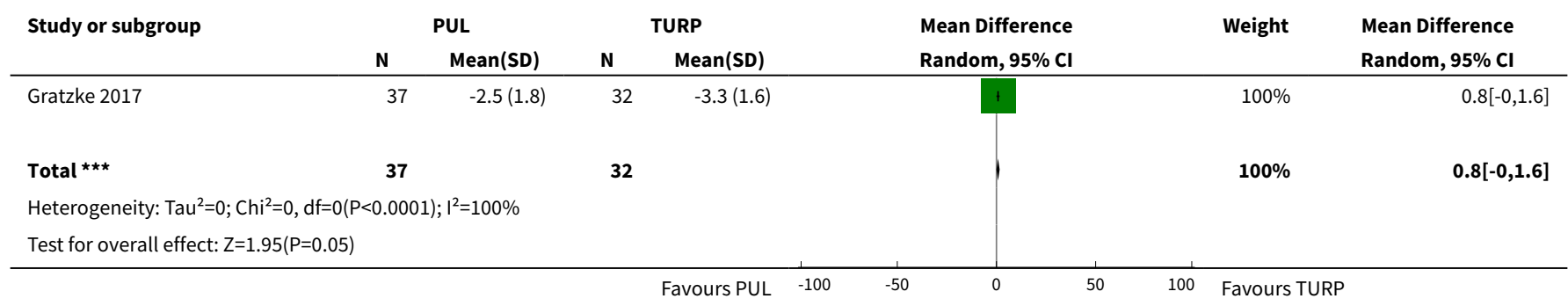

Analysis 3.3. Comparison 3 Prostatic urethral lift (PUL) versus transurethral resection of prostate (TURP) (long term), Outcome 3 Retreatment.

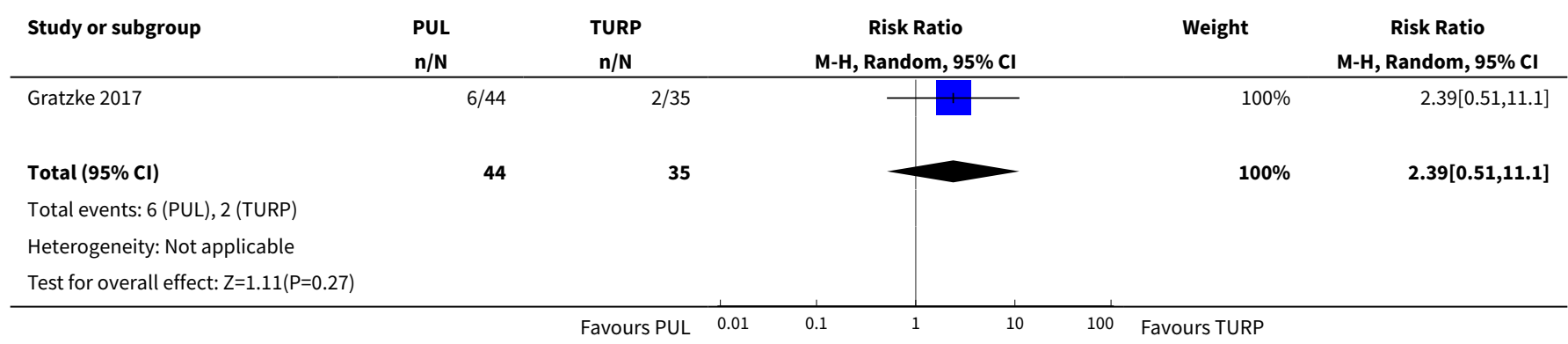

Analysis 3.4. Comparison 3 Prostatic urethral lift (PUL) versus transurethral resection of prostate (TURP) (long term), Outcome 4 Erectile function.

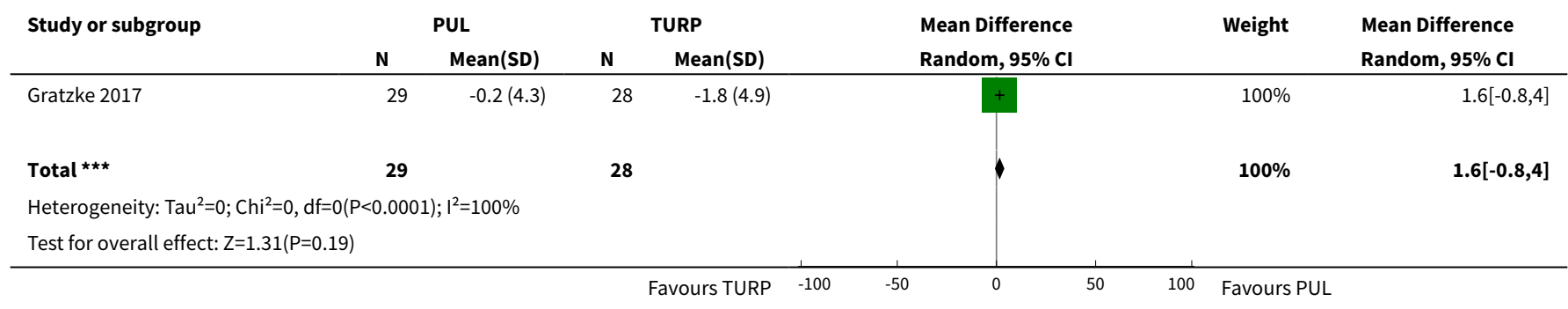


Analysis 3.5. Comparison 3 Prostatic urethral lift (PUL) versus transurethral resection of prostate (TURP) (long term), Outcome 5 Ejaculatory function.

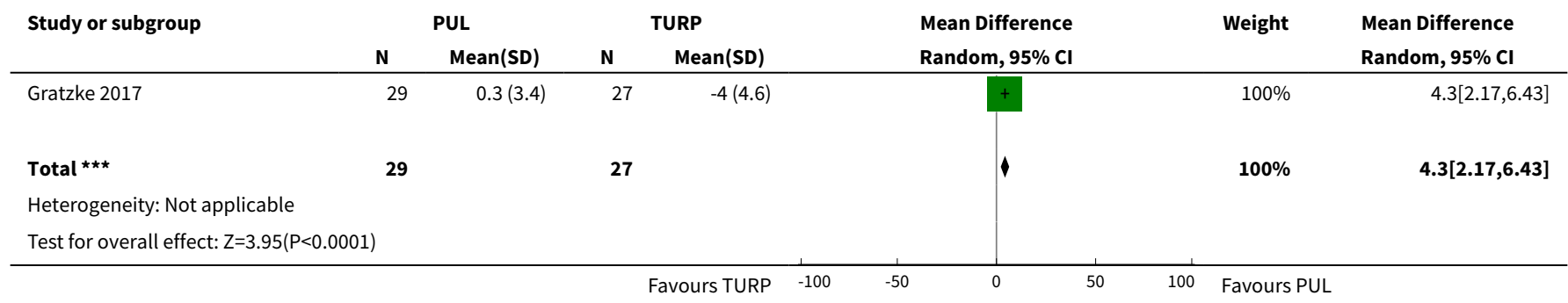

Analysis 3.6. Comparison 3 Prostatic urethral lift (PUL) versus transurethral resection of prostate (TURP) (long term), Outcome 6 Minor adverse events.






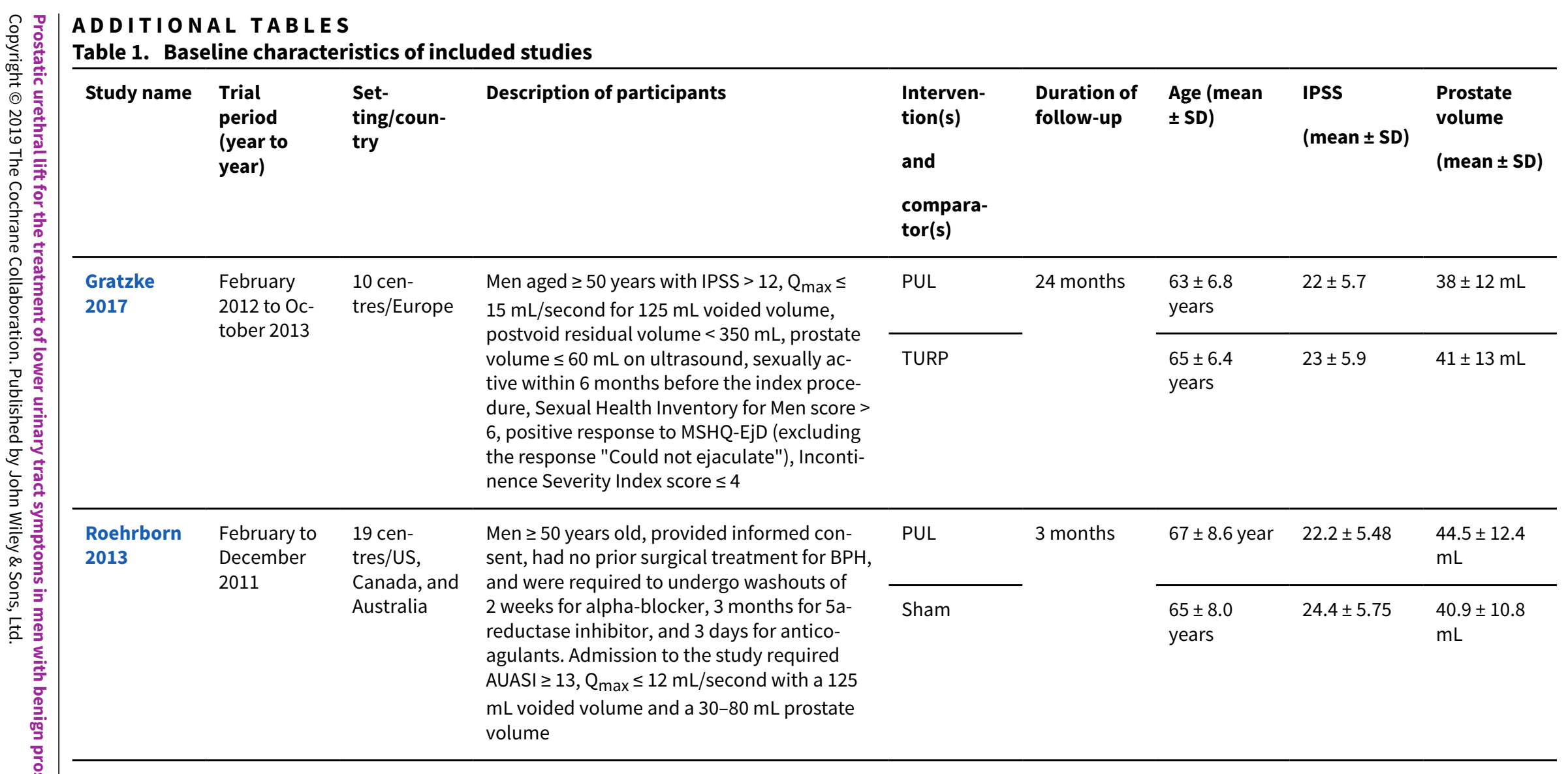

AUASI: American Urological Association Symptom Index; BPH: benign prostatic hyperplasia; IPSS: International Prostate Symptom Score; MSHQ-EjD: Male Sexual Health Questionnaire for Ejaculatory Dysfunction; PUL: prostatic urethral lift; $\mathrm{Q}_{\max }$ : maximum flow rate; TURP: transurethral resection of prostate. 
Table 2. Participants in included studies

\begin{tabular}{|c|c|c|c|c|c|c|}
\hline $\begin{array}{l}\text { Study } \\
\text { name }\end{array}$ & $\begin{array}{l}\text { Intervention(s) and com- } \\
\text { parator(s) }\end{array}$ & $\begin{array}{l}\text { Screened/ } \\
\text { eligible (n) }\end{array}$ & $\begin{array}{l}\text { Random- } \\
\text { ized (n) }\end{array}$ & $\begin{array}{l}\text { Analyzed } \\
\text { (n): effica- } \\
\text { cya }\end{array}$ & $\begin{array}{l}\text { Analyzed } \\
\text { (n): safe- } \\
\text { tyb }\end{array}$ & Finishing trial (n (\%)) \\
\hline \multirow{3}{*}{$\begin{array}{l}\text { Gratzke } \\
2017\end{array}$} & PUL & NR/91 & 45 & 37 & 45 & $44(97.7)$ \\
\hline & TURP & & 46 & 32 & 35 & $35(76.0)$ \\
\hline & Total & & 91 & 69 & 80 & $79(86.8)$ \\
\hline \multirow{3}{*}{$\begin{array}{l}\text { Roehrborn } \\
2013\end{array}$} & PUL & $430 / 206$ & 140 & 140 & 140 & $140(100.0)$ \\
\hline & Sham & & 66 & 66 & 66 & $66(100.0)$ \\
\hline & Total & & 206 & 206 & 206 & $206(100.0)$ \\
\hline \multirow{4}{*}{$\begin{array}{l}\text { Grand to- } \\
\text { tal }\end{array}$} & Intervention: PUL & - & 185 & 177 & 185 & $184(99.4)$ \\
\hline & Comparator: sham & & 66 & 66 & 66 & $66(100.0)$ \\
\hline & Comparator: TURP & & 46 & 32 & 35 & $35(76.0)$ \\
\hline & Overall & & 297 & 275 & 286 & $285(95.9)$ \\
\hline
\end{tabular}

n: number of participants; NR: not reported; PUL: prostatic urethral lift; TURP: transurethral resection of prostate.

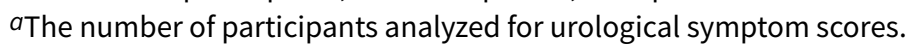

bThe number of participants with adverse events.

\section{AP PEN DICES}

\section{Appendix 1. Search strategy}

\section{Cochrane Library (via Wiley)}

$1 \mathrm{MeSH}$ descriptor: [Prostatic Hyperplasia] explode all trees

2 (prostat ${ }^{\star}$ near/3 hyperplasia*):ti,ab,kw (Word variations have been searched)

3 (prostat $^{\star}$ near/3 hypertroph $\left.{ }^{\star}\right): t i, a b, k w$ (Word variations have been searched)

4 (prostat $^{\star}$ near/3 adenoma*):ti,ab,kw (Word variations have been searched)

5 (BPH or BPO or BPE):ti,ab,kw (Word variations have been searched)

6 (prostat* near/3 enlarg*):ti,ab,kw (Word variations have been searched)

$7 \mathrm{MeSH}$ descriptor: [Prostatism] explode all trees

8 prostatism:ti,ab,kw (Word variations have been searched)

9 MeSH descriptor: [Urinary Bladder Neck Obstruction] explode all trees

10 ("bladder outlet obstruction" or BOO):ti,ab,kw (Word variations have been searched)

$11 \# 1$ or \#2 or \#3 or \#4 or \#5 or \#6 or \#7 or \#8 or \#9 or \#10

Prostatic urethral lift for the treatment of lower urinary tract symptoms in men with benign prostatic hyperplasia (Review) 
(Continued)

12 ("Prostatic urethral lift" or urolift):ti,ab,kw (Word variations have been searched)

$13 \# 11$ and \#12

\section{MEDLINE (via Ovid)}

1 exp Prostatic Hyperplasia/

2 (Prostat* adj3 hyperplasia*).tw.

3 (Prostat ${ }^{\star}$ adj3 hypertroph ${ }^{\star}$ ).tw.

4 (Prostat* ${ }^{\star}$ adj3 adenoma*).tw.

5 (BPH or BPO or BPE).tw.

6 (prostat $^{\star}$ adj3 enlarg*).tw.

7 exp Prostatism/

8 Prostatism.tw.

9 exp Urinary Bladder Neck Obstruction/

10 (Bladder $^{\star}$ adj3 obstruct ${ }^{\star}$ ).tw.

11 BOo.tw.

121 or 2 or 3 or 4 or 5 or 6 or 7 or 8 or 9 or 10 or 11

13 Prostatic urethral lift.tw.

14 UroLift.tw.

1513 or 14

1612 and 15

17 (animals not (humans and animals)).sh.

1816 not 17

\section{Embase (via Elsevier)}

1 'prostate hypertrophy'/exp

2 (Prostat ${ }^{\star}$ NEAR/3 hyperplasia*):ab,ti

3 (Prostat* NEAR/3 hypertroph *):ab,ti

4 (Prostat ${ }^{\star}$ NEAR/3 adenoma*):ab,ti

5 'bph':ab,ti OR 'bpo':ab,ti OR 'bpe':ab,ti

6 (prostat ${ }^{\star}$ NEAR/3 enlarg ${ }^{\star}$ ):ab,ti

7 'prostatism'/exp

8 'prostatism':ab,ti

9 'bladder obstruction'/exp

10 (bladder* NEAR/3 obstruct ${ }^{\star}$ ):ab,ti

11 'BOO':ab,ti 
(Continued)

12 \#1 OR \#2 OR \#3 OR \#4 OR \#5 OR \#6 OR \#7 OR \#8 OR \#9 OR \#10 OR \#11

13 'Prostatic urethral lift':ab,ti

14 'urolift':ab,ti

$15 \# 13$ OR \#14

16 \#12 AND \#15

17 ('animals'/exp) NOT ('humans'/exp and 'animals'/exp)

18 \#16 NOT \#17

\section{LILACS}

1 (mh:("Prostatic Hyperplasia" or Prostatism or "Urinary Bladder Neck Obstruction")) OR (tw:("Prostatic Hyperplasia" or "Prostatic Adenoma" or "Prostatic Hypertrophy" or "Prostatic Enlargement" or BPH or BPO or BPE or Prostatism or "Bladder Neck Obstruction" or "Bladder Outlet Obstruction" or BOO))

2 (tw:("Prostatic urethral lift" or urolift))

31 AND 2

\section{Scopus}

1 TITLE-ABS-KEY((hyperplasia* W/3 prostat $\left.{ }^{\star}\right)$ OR (hypertroph* W/3 prostat $\left.{ }^{\star}\right)$ OR (adenoma* W/3 prostat ${ }^{\star}$ ) OR (prostat ${ }^{\star}$ W/3 enlarg ${ }^{\star}$ ) OR (bph OR bpo OR bpe OR boo) OR prostatism OR (bladder ${ }^{\star} W / 3$ obstruct $\left.^{\star}\right)$ )

2 TITLE-ABS-KEY("Prostatic urethral lift" or UroLift)

31 AND 2

\section{Web of Science}

$1 \mathrm{TS}=\left(\left(\right.\right.$ hyperplasia* NEAR/3 prostat $\left.{ }^{\star}\right)$ OR (hypertroph* NEAR/3 prostat $\left.{ }^{\star}\right)$ OR (adenoma* NEAR/3 prostat ${ }^{\star}$ ) OR (prostat* NEAR/3 enlarg*) OR (bph OR bpo OR bpe OR boo) OR prostatism OR (bladder* NEAR/3 obstruct*))

2 TS= ("Prostatic urethral lift" or UroLift)

31 AND 2

\section{Google Scholar}

1 allintitle: ("Prostatic Hyperplasia" OR "prostatic hypertrophy" OR prostatism OR "bladder obstruction" OR "bladder outlet obstruction" OR bph OR bpo OR bpe OR boo) AND ("Prostatic urethral lift" OR UroLift))

\section{ClinicalTrials.gov}

1 ("Prostatic Hyperplasia" OR "Prostatic Hypertrophy" OR "Prostatic Adenoma" OR BPH OR BPO OR BPE OR Prostatism OR "Bladder Neck Obstruction" OR "Bladder Outlet Obstruction" OR BOO)

2 ("Prostatic urethral lift" OR urolift)

31 AND 2

\section{World Health Organization International Clinical Trials Registry Platform Search Portal}

1 In the title = ("Prostatic Hyperplasia" OR "Prostatic Hypertrophy" OR "Prostatic Adenoma" OR BPH or BPO or BPE OR Prostatism OR "Bladder Neck Obstruction" or "Bladder Outlet Obstruction" or BOO) AND In the intervention= ("Prostatic urethral lift" or urolift) 
(Continued)

\section{Grey Literature (Open Grey)}

1 ("Prostatic Hyperplasia" OR "Prostatic Hypertrophy" OR "Prostatic Adenoma" OR BPH or BPO or BPE OR Prostatism OR "Bladder Neck Obstruction" or "Bladder Outlet Obstruction" or BOO)

2 ("Prostatic urethral lift" or urolift OR "Urethra")

31 AND 2

Appendix 2. Survey of trial investigators providing information on included trials

\begin{tabular}{llll}
\hline Study & $\begin{array}{l}\text { Date trial author } \\
\text { contacted (first) }\end{array}$ & $\begin{array}{l}\text { Date trial author } \\
\text { provided data (lat- } \\
\text { est) }\end{array}$ & $\begin{array}{l}\text { Data trial author provided } \\
\text { (short summary) }\end{array}$ \\
\hline Roehrborn 2013 & 1 November 2017 & 20 May 2018 & $\begin{array}{l}\text { Hospital stay and allocation concealment from additional pub- } \\
\text { lications/ Blinding (performance bias and detection bias) }\end{array}$ \\
\hline
\end{tabular}

\section{CONTRIBUTIONS OF AUTHORS}

$\mathrm{JHJ}$ : conception and study design, drafting the protocol, searching for trials, study selection, extracting data, assessing risk of bias, performing data analysis, interpretation of data, and drafting the review.

BR: drafting the protocol, searching for trials, study selection, extracting data, assessing risk of bias, performing data analysis, and drafting the review.

KAM: drafting the protocol, searching for trials, study selection, and drafting the review.

MB: drafting the protocol, and providing clinical and methodological advices on the review.

VN: drafting the protocol, and providing clinical and methodological advices on the review.

MHK: creating search strategies and searching for trials.

PD: conception and study design, providing clinical and methodological advice on the review, and final approval.

\section{DECLARATIONSOF INTEREST}

$\mathrm{JHJ}$ : none.

BR: none.

KAM: none.

MB: Boston Scientific (consultant for endourology and stone management), Auris Health (consultant for robotic surgery and endourology).

VN: none.

MHK: none.

PD: none.

\section{SOURCES OF SUPPORT}

\section{Internal sources}

- Department of Urology, Yonsei University Wonju College of Medicine, Korea, South.

- Minneapolis VA Health Care System, USA.

Prostatic urethral lift for the treatment of lower urinary tract symptoms in men with benign prostatic hyperplasia (Review) 
- Department of Urology, University of Minnesota, USA.

\section{External sources}

- No sources of support supplied

\section{DIFFERENCES BETWEEN PROTOCOLANDREVIEW}

This review was based on a published protocol with differences as described here (Jung 2017).

- While we planned to include non-RCTs (see 'Types of studies'), no studies were found. Therefore, the planned methods for non-RCTs were not implemented in the review due to lack of data.

- Types of outcome measures: we used a minimal clinically important difference of 0.5 to assess quality of life based on a newly cited reference (Rees 2015). We analyzed retreatment outcomes for short term (12 months or less) and long term (greater than 12 months) separately.

- Types of outcome measures: we used proportion of participants with an indwelling catheter at postoperative 24 hours as a secondary outcome due to lack of data.

- Electronic searches: we additionally have searched Google scholar.

\section{NOTES}

We based parts of the Methods section of this review on a standard template developed by Cochrane Metabolic and Endocrine Disorders Group, which was modified and adapted for use by Cochrane Urology.

\section{INDEX TERMS}

\section{Medical Subject Headings (MeSH)}

Lower Urinary Tract Symptoms [ ${ }^{*}$ etiology]; Prostatic Hyperplasia [ ${ }^{*}$ complications]; Transurethral Resection of Prostate; Urethra [physiopathology] [*'surgery]

\section{MeSH check words}

Aged; Humans; Male 